\title{
Atomic Embeddability, Clustered Planarity, and Thickenability*
}

\author{
Radoslav Fulek ${ }^{\dagger} \quad$ Csaba D. Tóth ${ }^{\ddagger}$
}

\begin{abstract}
We study the atomic embeddability testing problem, which is a common generalization of clustered planarity (cplanarity, for short) and thickenability testing, and present a polynomial time algorithm for this problem, thereby giving the first polynomial time algorithm for c-planarity.

C-planarity was introduced in 1995 by Feng, Cohen, and Eades as a variant of graph planarity, in which the vertex set of the input graph is endowed with a hierarchical clustering and we seek an embedding (crossing free drawing) of the graph in the plane that respects the clustering in a certain natural sense. Until now, it has been an open problem whether c-planarity can be tested efficiently, despite relentless efforts. The thickenability problem for simplicial complexes emerged in the topology of manifolds in the 1960s. A 2-dimensional simplicial complex is thickenable if it embeds in some orientable 3-dimensional manifold. Recently, Carmesin announced that thickenability can be tested in polynomial time.

Our algorithm for atomic embeddability combines ideas from Carmesin's work with algorithmic tools previously developed for weak embeddability testing. We express our results purely in terms of graphs on surfaces, and rely on the machinery of topological graph theory.

Finally we give a polynomial-time reduction from cplanarity to thickenability and show that a slight generalization of atomic embeddability to the setting in which clusters are toroidal graphs is NP-complete.
\end{abstract}

\section{Introduction}

Clustered planarity (for short, c-planarity) was introduced in 1995 by Feng, Cohen, and Eades [22. 23. motivated by applications in set visualization. Lengauer 32] considered one of its variants already in the 1980s. The problem can be seen as a hierarchical variant of planarity testing; a problem for which a linear-time algorithm has been known for a long time [31. In the extensive literature devoted to cplanarity and its variants, the complexity status of only restricted special cases has been established, most notably in [2, 5, 17, 28, see also the somewhat outdated survey [16]. The c-planarity problem is formally stated as follows.

\footnotetext{
${ }^{*}$ Research supported in part by Austrian Science Fund (FWF): M2281-N35, and the National Science Foundation awards CCF1422311, CCF-1423615, and DMS-1800734.

$\dagger$ Department of Computer Science, University of Arizona, Tucson, AZ, USA

${ }^{\ddagger}$ Department of Mathematics, California State University Northridge, Los Angeles, CA, USA and Department of Computer Science, Tufts University, Medford, MA, USA.
}

Problem 1. (C-PlanARITy) We are given a simple graph $G=(V, E) ;$ a collection $\mathcal{C}$ of pairwise disjoint simple closed curves in $\mathbb{R}^{2}$; and a map $\mu: V \rightarrow \mathcal{R}$, where $\mathcal{R}$ is the set of path connected components (called regions) of $\mathbb{R}^{2} \backslash \bigcup_{C \in \mathcal{C}} C$. Decide whether there exists an embedding $\psi$ of $G$ in $\mathbb{R}^{2}$ such that $\psi(v) \in \mu(v)$ and $|\psi(e) \cap C| \leq 1$ for every $C \in \mathcal{C}$ and every edge $e \in E$.

Embeddability in $\mathbb{R}^{3}$ and thickenability. Note that a graph is a 1-dimensional simplicial complex. We consider the 2-dimensional analog of graph embeddings in $\mathbb{R}^{3}$. It is a well-known result that for every graph $G$ there exists an orientable surface (an orientable 2dimensional manifold) $S$ such that $G$ embeds in $S$. An analogous result fails for 2-dimensional polyhedra (2polyhedra for short) and 3-dimensional manifolds (3manifolds for short). A 2-polyhedron $P$ is thickenable if $P$ embeds 1 in some orientable 3 -manifold. It was known at least since the 1960s that testing whether a 2-polyhedron is thickenable is in NP, which is an immediate consequence of a theorem by Neuwirth [37]; see also 41. We restate it as Theorem 4.2 in Section 4 (in essence, it characterizes thickenability in terms of socalled link graphs). We remark that Neuwirth's theorem has been recently used in [34] in the first step of an algorithm that decides (not necessarily in polynomial time) whether a given 2-polyhedron embeds to $\mathbb{R}^{3}$.

The thickenability problem is formulated as follows. Let $H$ be a finite multigraph without loops (multiple edges are allowed). Let $P=(H, F)$ denote a 2dimensional (abstract) polyhedron, where $F$ is a set of cycles in $H$. We assume that every edge of $H$ is contained in at least one element of $F$. The multigraph $H$ is the 1-skeleton of $P$ and every element of $F$ is a facet in $P$. Note that $H$ might contain a cycle that is not a facet of $P$.

Problem 2. (Thickenability) Given a 2-polyhedron $P=(H, F)$, where $H$ is a multigraph without loops and $F$ is the set of facets of $P$, decide whether $P$ embeds to some orientable 3-manifold.

Recently, Carmesin [14, Section 6] announced that one can test whether a simply connected 2-polyhedron

\footnotetext{
${ }^{1}$ In this case, topological and piecewise linear embeddability are equivalent.
} 
embeds in $\mathbb{R}^{3}$ in quadratic time, while embeddability testing for general 2-polyhedra in $\mathbb{R}^{3}$ is known to be NPhard [20]. In the case of simply connected 2-polyhedra, thickenability is equivalent to embeddability in $\mathbb{R}^{3}$, see for example [13. Though this equivalence appears to be a well-known consequence of Perelman's famous result.

In fact, Carmesin's approach deals exclusively with testing the thickenability condition in Theorem 4.2 (cf. Section 4 ). Therefore his approach to the embeddability in $\mathbb{R}^{3}$ applies also to THICKENABILITY in general, but it is restricted simply connected 2-polyhedra. In contrast, testing whether a given 2-polyhedron, that is homeomorphic to a nonorientable surface, embeds in a given 3-manifold (that is, both the 2-polyhedron and the 3-manifold are given), is already NP-hard [12.

Atomic embeddibility was introduced in 2 and [25, Section 11], see also [26, as a common generalization of C-PLANARITY and THICKENABILITY. It is an extension of the concept of weak embeddability 2 (also known in topology as approximating simplicial maps by embeddings [36, 38, 40, 42]). We do not define weak embedding here, but remark that its study in computational geometry was motivated by the special case of a (piecewise linear) weak embeddings of a cycle in the plane, which corresponds to weakly simple polygons [1, 15, 18.

Let $G$ and $H$ be finite multigraphs without loops. To distinguish between $G$ and $H$ in our terminology, the vertices and edges of $H$ are called atoms and pipes, respectively. A map $\varphi: G \rightarrow H$ is simplicial if it maps vertices to vertices (i.e., to atoms), edges to vertices or edges (i.e., to atoms or pipes), and preserves edge-vertex incidences. An instance of atomic embeddability is given by a simplicial map $\varphi: G \rightarrow H$.

The thickening $\mathcal{H}$ of $H$ is an orientable 2dimensional surface constructed as follows. For each atom $\nu \in V(H)$, let $\mathcal{S}(\nu)$ be a 2 -sphere with $\operatorname{deg}(\nu)$ pairwise disjoint open discs, called holes, removed. We fix an orientation on $\mathcal{S}(\nu)$, and define an arbitrary oneto-one correspondence between the holes of $\mathcal{S}(\nu)$ and the pipes incident to $\nu$. The thickening $\mathcal{H}$ is obtained by gluing the surfaces $\mathcal{S}(\nu), \nu \in V(H)$, as follows; see Fig. 1 (left) for an illustration. For every pipe $\rho \in E(H)$, $\rho=\nu \mu$, identify the pair of boundaries of the holes corresponding to $\rho$ by an orientation reversing homeomorphism. In particular, if $\nu \mu \notin E(H)$, then $\mathcal{S}(\nu)$ and $\mathcal{S}(\mu)$ are disjoint ${ }^{2}$

An embedding $\mathcal{E}: G \rightarrow \mathcal{H}$ is an atomic embedding of $G$ with respect to $\varphi$ if every vertex $v \in V(G)$ is embedded in $\mathcal{S}(\varphi(v))$; and every edge $u v \in E(G)$ is

\footnotetext{
${ }^{2}$ The surface $\mathcal{H}$ is reminiscent of the ball-and-stick or spacefilling models in molecular chemistry.
}

embedded as a Jordan arc in $\mathcal{S}(\varphi(u)) \cup \mathcal{S}(\varphi(v))$ as follows: If $\varphi(u) \neq \varphi(v)$ then the Jordan arc representing $u v$ intersects the hole corresponding to the pipe $\varphi(u v)$ in exactly one point, which is a proper crossing, or in other words, a transversal intersection.

Problem 3. (Atomic embeddability) Given a pair of multigraphs without loops, $G$ and $H$, and a simplicial $\operatorname{map} \varphi: G \rightarrow H$, decide whether an atomic embedding of $G$ with respect to $\varphi$ exists.

We remark that an instance $(H, F)$ of THICKENABILITY corresponds to an instance $(G, H)$ of ATOMIC EMBEDDABILITY, where $H$ is the same graph both instances, and $G$ is a vertex disjoint union of cycles (disjoint copies of the cycles in $F$ ).

Results. In this paper, we present a polynomialtime algorithm for atomic embeddability, thereby giving the first polynomial-time algorithm for c-planarity. Our approach combines ideas from Carmesin's work 14 with algorithmic tools previously developed for weak embeddability testing. In particular, the elementary operation "stretch" (defined below) is based on a similar operation in 14. However, by formulating the problem in terms of graphs on surfaces, our results are more general and perhaps more accessible to the broader community. A polynomial-time algorithm for c-planarity implies that some other constrained planarity problems that have previously been reduced to c-planarity are tractable, as well; see 3 and [4, Figure 4].

We also consider a further generalization of ATOMIC EMBEDDABILITY in which the surfaces $\mathcal{S}(\nu), \nu \in V(H)$, may have higher genus (by attaching additional handles), and show that this problem is NP-complete even if each surface $\mathcal{S}(\nu)$ is based on a torus rather than a sphere.

Finally, we give a short polynomial-time reduction of C-PLANARITY to THICKENABILITY. By combinining the results of Schaefer [39, Theorem 6.17], and Angelini and Da Lozzo 4 we observe that C-PLANARITY is polynomial-time equivalent to CONNECTED SEFE- 2 , the problem of deciding simultaneous embeddability of two graphs in the case when the intersection of the two graphs is connected (see Section 4 for a formal statement of the problem). The general version of the problem, known as SEFE-2, where the intersection of the two graphs may be disconnected, is notoriously difficult. Introduced by Brass et al. [10, it subsumes most of the studied planarity variants [39, Figure 2], and has generated considerable research activity [3, 7, 9, 29]; see also 8 for a survey. Therefore it is an unfortunate state of affairs that its complexity status is still unknown. Although, Carmesin's and our results give a hope that a resolution of SEFE-2 problem might be within reach. 


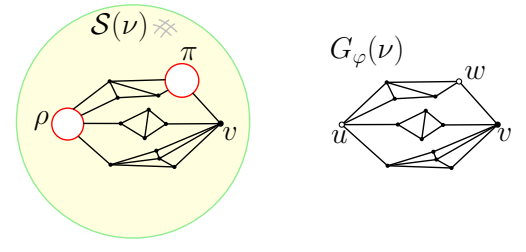

Figure 1: Part of an atomic embedding of $G$ on $\mathcal{S}(\nu)$, where the atom $\nu$ is incident to pipes $\rho$ and $\pi$ (left), and the corresponding local graph $G_{\varphi}(\nu)$ (right). The virtual vertices $u$ and $w$ in $G_{\varphi}(\nu)$ correspond to the pipes $\rho$ and $\pi$, respectively. All other vertices in $G_{\varphi}(\nu)$ are ordinary.

Let us note that there also exists a natural and fairly straightforward polynomial-time reduction of $\mathrm{CON}$ NECTED SEFE-2 to THICKENABILITY, which was found independently by de Mesmay, Kaluža, and Tancer 11 35] and these authors. This suggests that THICKENABILITY, and hence, ATOMIC EMBEDDABILITY, is not powerful enough to solve SEFE-2 in general without using significantly novel ideas.

Organization. Section 2 presents a polynomialtime algorithm for atomic embeddability. Section 3 shows that a further generalization of the problem is NP-hard. In Section 4, we give a direct polynomialtime reduction of C-PLANARITY to THICKENABILITY, which also establishes a polynomial reduction of another problem, CONNECTED SEFE-2, to THICKENABILITY.

\section{Atomic Embeddings}

In this section we present a polynomial-time algorithm for ATOMIC EMBEDDABILITY. After defining local graphs, which are crucial for the algorithm, we present a high-level overview in Section 2.1. Section 2.2 introduces additional terminology. We reduce a given instance $\varphi$ to normal form (defined below) in Section 2.3 and introduce five elementary operations on atomic instances in Section 2.4 that are used in our main algorithm. We show how to solve two special cases in linear time in Sections 2.5 and 2.6 Our main algorithm in Section 2.7 reduces all normal instances to these special cases. We finish with a running time analysis in Section 2.8 .

Local graphs. Let $\varphi: G \rightarrow H$ be an instance of atomic embeddability. The simplicial map $\varphi: G \rightarrow H$ naturally extends to subgraphs of $G$. For an atom $\nu \in V(H), \varphi^{-1}[\nu]$ denotes the subgraph of $G$ mapped to $\nu$ by $\varphi$. For a pipe $\rho \in E(H), \varphi^{-1}[\rho]$ denotes the subset of edges of $G$ mapped to $\rho$ by $\varphi$.

For every atom $\nu \in V(H)$, we define a multigraph $G_{\varphi}(\nu)$, which captures the local structure of $\varphi$ at the atom $\nu$ and its incident pipes; see Fig. 1 for an illustration. (We remark that graphs $G_{\varphi}(\nu)$ are analogous to the graphs $\bar{C}$ in [2] and the links in [13].)

The vertices of $G_{\varphi}(\nu)$ are in a one-to-one correspondence with the union of the set of vertices in $V(G)$ mapped by $\varphi$ to $\nu$ (that is, $V\left(\varphi^{-1}[\nu]\right)$ ) and the set of pipes incident to $\nu$. Hence, we can distinguish between ordinary vertices that correspond to vertices in $V\left(\varphi^{-1}[\nu]\right)$ and virtual vertices that correspond to pipes incident to $\nu$. For every edge in $E(G)$ between two vertices in $V\left(\varphi^{-1}[\nu]\right)$ in $G$, add an edge in $G_{\varphi}(\nu)$ between the corresponding vertices. Finally, for every edge in $u v \in E(G)$ where $u \in V\left(\varphi^{-1}[\nu]\right)$ and $v \notin V\left(\varphi^{-1}[\nu]\right)$, add an edge $G_{\varphi}(\nu)$ between the ordinary vertex $u$ and the virtual vertex corresponding to $\varphi(u v)$. Thus, edges of $G_{\varphi}(\nu)$ are in a one-to-one correspondence with the union of the edges of $G$ between vertices in $V\left(\varphi^{-1}[\nu]\right)$ and the edges of $G$ mapped to pipes incident to $\nu$ by $\varphi$. Let $\bar{e} \in E(G)$ denote the edge corresponding to an edge $e \in E\left(G_{\varphi}(\nu)\right)$.

Note that the virtual vertices form an independent set in $G_{\varphi}(\nu)$. An embedding $\mathcal{E}_{\nu}$ of $G_{\varphi}(\nu)$ is inherited from an atomic embedding $\mathcal{E}$ of $G$, if $\mathcal{E}_{\nu}$ is obtained from the restriction $\mathcal{E}$ to $\mathcal{S}(\nu)$ by filling the holes of $\mathcal{S}(\nu)$ with discs, and then contracting them to points.

Let $\mathcal{E}: G \rightarrow \mathcal{S}$ be an embedding of a graph on an orientable surface. The rotation at a vertex $v \in V(G)$ is the counterclockwise cyclic order of the end pieces of the edges incident to $v$. The rotation system of $\mathcal{E}$ is the set of rotations of all vertices of $G$. A vertex $v$ of a planar graph has a fixed rotation (for short, is fixed) if its rotation in every embedding of the graph in the plane is unique up to the choice of orientation. For a pair of virtual vertices $u$ and $v$ of $G_{\varphi}(\mu)$ and $G_{\varphi}(\nu)$, resp., corresponding to a pipe $\mu \nu=\rho \in E(H)$, the edges $e$ incident to $u$ (resp., $v$ ) correspond to edges $\bar{e} \in \varphi^{-1}[\rho]$. The rotations of $u$ and $v$ in an embedding of all local graphs in the plane determine two cyclic orders $\varphi^{-1}[\rho]$. This allows us, in particular, to define that the rotations at $u$ and $v$ to be opposite (in other words, reverse) to each other, if the rotation at $u$ is $\left(u u_{1}, \ldots, u u_{\operatorname{deg}(u)}\right)$, at $v$ it is $\left(v v_{\operatorname{deg}(v)}, \ldots, v v_{1}\right)$, and $\overline{u u_{i}}=\overline{v v_{i}}=u_{i} v_{i} \in E(G)$. The rotations of $u$ and $v$ are compatible if they are the same or opposite to each other; and incompatible otherwise.

An instance $\varphi$ of ATOMIC EMBEDDIBILITY is positive if there exists an atomic embedding of $G$ with respect to $\varphi$. Two instances, $\varphi$ and $\varphi^{\prime}$, are equivalent if $\varphi$ and $\varphi^{\prime}$ are both positive, or $\varphi$ and $\varphi^{\prime}$ are both negative. We can now formulate ATOMIC EMBEDDIBILITY in terms of the rotation systems of plane embeddings of the graphs $G_{\varphi}(\nu), \nu \in V(H)$.

Observation 1. An instance $\varphi: G \rightarrow H$ of atomic 
embeddability is positive if and only if the graphs $G_{\varphi}(\nu)$, $\nu \in V(H)$, are planar, and they each have embeddings in the plane such that for every pipe $\mu \nu \in E(H)$, the virtual vertices corresponding to $\mu \nu$ in $G_{\varphi}(\mu)$ and $G_{\varphi}(\nu)$ have opposite rotations (in the sense that we consider every edge e incident to a virtual vertex as $\bar{e}$ ).

For a subset $V^{\prime} \subset V(G)$, a $V^{\prime}$-bridge $B$ in $G$ is a subgraph of $G$ obtained as the union of $V^{\prime}$, a connected component $C$ of $G \backslash V^{\prime}$, and all the edges joining a vertex of $C$ with a vertex of $V^{\prime}$. We allow $B$ to consist of a single edge between two vertices in $V^{\prime}$, or a loop incident to a vertex in $V^{\prime}$ (see Fig. 2(left)). Analogously to Carmesin [13], we also define two special graphs (as possible local graphs). A p-path is a graph that consists of two vertices (poles) connected by one or more subdivided edges (Fig. 2(middle)). A p-star is a graph with a unique cut vertex (center) whose bridges are p-paths with one pole at the center (Fig. 2(right)).

\subsection{High Level Overview of the Recognition} Algorithm Given an instance $\varphi$ of atomic embeddiblity, we apply a sequence of elementary operations that each produces an equivalent instance $\varphi^{\prime}$ (with respect to atomic embeddability). Intermediate steps of our algorithm may detect that the instance is negative when a local graph $G_{\varphi}(\nu)$ is nonplanar. It may also disconnect the graph $H$, effectively splitting an instance into independent instances. Ultimately, it reduces $\varphi$ to a family of instances, each of which is either toroidal (where both $G$ and $H$ are 2-regular), or subcubic (where the maximum degree of all local graphs is at most 3). In both cases, we can easily test atomic embeddibility in linear time (Sections 2.5 and 2.6). Hence, the witness of atomic non-embeddibility that is provided by our algorithm is either the non-planarity of a local graph in an instance produced by a sequence of elementary operations, or negative subcubic or toroidal instance.

Let $\mathcal{G}^{*}$ denote the disjoint union of all local graphs except those belonging to toroidal subinstances. Our algorithm incrementally reduces the maximum degree $\Delta=\Delta(\varphi)=\max _{v \in V\left(\mathcal{G}^{*}\right)} \operatorname{deg}(v)$. The two key operations for dealing with a vertex $v \in V\left(\mathcal{G}^{*}\right)$ of degree $\Delta \geq 4$ are $\operatorname{Stretch}(v,$.$) , which splits v$ into two vertices of smaller degree (illustrated in Fig. 7), and Contract(.), which contracts a pipe (illustrated in Fig. 11). Operation Stretch(.) can be applied to a virtual or an ordinary vertex: If it is applied to an ordinary vertex, it modifies only $G$ and not $H$, but if it is applied to a virtual vertex, it modifies both $G$ and $H$, and in particular it increases the genus of the surface $\mathcal{H}$ by 1 . We note that the increase in the genus of $\mathcal{H}$ occurs also in the special case that $\varphi$ represents an instance of c-planarity (when $\mathcal{H}$ is initially homeomorphic to a 2 -sphere, i.e., when its genus is 0 ). This explains in part why this approach for the inherently planar problem of c-planarity has not been considered before. The generalization of cplanarity to surfaces of higher genus allows for a broader range of operations, but it also poses several technical challenges that had to be resolved - some of them even indicated that the problem might be NP-complete, which we discuss next.

Unfortunately, Stretch $(v,$.$) produces an equivalent$ instance only if we already have some partial information about the rotation of vertex $v$. In general, it cannot reduce the degree of a cut vertex. This obstacle is overcome with the help of a surprisingly simple operation, Contract $(\rho)$, which contracts a pair of atoms in $H$ joined by a single pipe $\rho$ into one atom, thereby eliminating a pair of virtual vertices in $\mathcal{G}^{*}$ corresponding to $\rho$. An almost identical operation is also crucial in our recent joint work with Akitaya 2 about weak embeddability. Nevertheless, the possibility of using this operation in the context of (the general case of) c-planarity or atomic embeddability was not clear to us for some time. The reason is that the operation $\operatorname{Contract}(\rho)$ for a pipe $\rho=\mu \nu$ can only be applied in a very restricted setting, essentially if and only if $G_{\varphi}(\mu)$ or $G_{\varphi}(\nu)$ is a p-path and $\rho$ corresponds to a pole of that p-path; or if they are both p-stars and $\rho$ corresponds to their centers. The crucial observation that saves the day, which is implicit in Carmesin's work, is that after some preprocessing that resolves 2-cuts with a vertex of degree $\Delta$, we can use the operation Enclose(.), illustrated in Fig. 6. to turn each cut vertex of degree $\Delta$ into a center of a p-star.

In order to show that our algorithm runs in polynomial time, we define a nonnegative potential $\Phi(\varphi)$ bounded from above throughout the execution of the algorithm by a polynomial function of $|V(G)|$ that strictly decreases after every application of Stretch(.) or Contract(.), but unfortunately, not after every application of Enclose(.), which possibly just creates a pair of new virtual vertices in $\mathcal{G}^{*}$. Hence, we had to design a charging scheme that controls the growth of $\mathcal{G}^{*}$.

Several other similar, but less crucial, operations are used in the preprocessing and postprocessing steps of the algorithm, where the preprocessing step normalizes the input instance in order to allow a relatively smooth runtime analysis, and the postprocessing step handles toroidal instances and subcubic instances (where $\Delta(\varphi) \leq 3$ ).

2.2 Preliminaries Let $G$ and $H$ be multigraphs without loops (multiple edges are allowed in both $H$ and $G)$. By a slight abuse of notation, if there is no danger of confusion, we sometimes denote edges by unordered 
pairs of their endpoints (even though several edges may connect the same pair of vertices). A path, cycle, and walk in a graph is always assumed to be a sequence of edges (rather than vertices). Recall that in order to distinguish $G$ and $H$ in our terminology, the vertices and edges of $H$ are called atoms and pipes, respectively. We use the convention that vertices and edges of $G$ are denoted by lower case Roman letters (e.g., $u, v, z$ and $e, f, g)$, respectively, and the atoms and pipes by lower case Greek letters (e.g., $\nu, \mu$ and $\rho, \pi$ ).

Cut vertices, 2-cuts, and 2-edge-cuts. Every vertex of degree 2 or less has a unique rotation, hence it has a fixed rotation. For this reason, we use a topological notion of 1- and 2-cuts, which is invariant to subdivisions of edges and supression of vertices of degree 2 . For a connected graph $G$, which is not a cycle, denote by $G^{-}$the multigraph obtained by supressing all vertices of degree 2. Hence, $G^{-}$is free of subdivided edges, defined as paths whose internal vertices have degree 2. Note that $G^{-}$can have loops corresponding to cycles in $G$ that form leaf blocks.

Let $G$ be a connected graph that is not a cycle. A vertex $v \in V\left(G^{-}\right) \subseteq V(G)$ is a proper cut vertex (or proper 1-cut) of $G$ if there are two or more $\{v\}$-bridges in $G^{-}$. A pair of vertices $\{u, v\} \subset V\left(G^{-}\right) \subseteq V(G)$ is a proper 2-cut of $G$ if there are at least three $\{u, v\}$ bridges in $G^{-}$, or there are exactly two $\{u, v\}$-bridges in $G^{-}$, neither of which is an edge in $G^{-}$. (Note that if there are exactly two $\{u, v\}$-bridges in $G^{-}$, and one of them is an edge between $u$ and $v$, then $\{u, v\}$ is not a 2-cut in $G^{-}$.) A pair of edges $\{e, f\} \subset E(G)$, such that at least one vertex incident to $e$ and one vertex incident to $f$ is of degree at least 3 , is a proper 2-edgecut of $G$ if there exist edges $e^{-}$and $f^{-}$, such that $e^{-}$ and $f^{-}$were obtained by suppressing internal vertices of degree 2 of a path containing $e$ and $f$, respectively, and $\left\{e^{-}, f^{-}\right\}$is a 2-edge-cut in $G^{-}$. Finally, for a proper 2-cut $\{u, v\}$, a $\{u, v\}$-bridge $B$ is separable if $\operatorname{deg}_{B}(u) \in\{1, \operatorname{deg}(u)-1\}$ and $\operatorname{deg}_{B}(v) \in\{1, \operatorname{deg}(v)-1\}$, otherwise it is nonseparable.

OBSERVATION 2. Let $G$ be a connected graph that is not a cycle. If $\{u, v\}$ is a proper 2-cut and $B$ is a separable $\{u, v\}$-bridge but not a subdivided edge, then there exists a proper 2-edge-cut $\{e, f\}$ in $G$ such that $u \in e$ and $v \in f$.

We often tacitly use the following well-known result by Mac Lane 33. If $G$ is a connected planar graph, and the rotation of a vertex $v$ is not fixed, then $\operatorname{deg}(v) \geq 3$ and $v$ participates in a proper 1- or 2-cut. In particular, if every graph $G_{\varphi}(\nu), \nu \in V(H)$, is a subdivision of a 3connected graph, we can use planarity testing to check the conditions in Observation 1, and easily reduce the

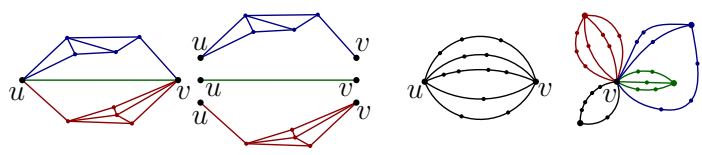

Figure 2: A proper 2-cut $\{u, v\}$ and its three bridges (left), a p-path with poles $u$ and $v$ (middle), and a pstar centered at $v$ (right).

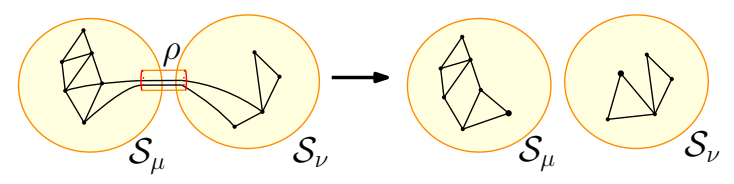

Figure 3: An atomic embedding of $G$ on $\mathcal{S}(\mu) \cup \mathcal{S}(\nu)$ before and after operation Suppress $(\rho)$, where $\rho=\mu \nu$. The operation eliminates a pipe with at most two edges of $G$.

atomic embedibility problem to 2SAT (cf. Section 2.6). The challenge is, therefore, to handle the possible rotations of vertices that participate in proper 1- or 2cuts in some local graph $G_{\varphi}(\nu)$.

2.3 Preprocessing and Data Structures Our algorithm uses a sequence of elementary operations that dynamically modify a given instance $\varphi: G \rightarrow H$ of atomic embeddability. For the running time analysis (Section 2.8), we need to maintain data structures that support these operations. We assume that the input specifies $G, H$, and $\varphi$ explicitly (i.e., adjacency lists for the graphs $G$ and $H$, and pointers from the vertices and edges of $G$ to their images in $H$ under the map $\varphi: G \rightarrow H)$. The size of an instance $\varphi: G \rightarrow H$ is the total number of edges and vertices in the graphs $G$ and $H$. Before we present our data structures (which do not maintain $H$ and $\varphi$ explicitly), we preprocess the instance $\varphi$.

Definition 1. An instance $\varphi: G \rightarrow H$ of atomic embeddability is normal if

- the degree of every virtual vertex in every $G_{\varphi}(\nu)$, $\nu \in V(H)$, is 3 or higher; and

- $G_{\varphi}(\nu)$ is connected for all $\nu \in V(H)$.

We define an operation that eliminates pipes with 2 or less edges, see Fig. 3

Suppress $(\rho)$. We are given a pipe $\rho \in E(H)$ such that $\left|\varphi^{-1}[\rho]\right| \leq 2$. Let $\mu, \nu \in V(H)$ be the two atoms incident to $\rho$. Remove the pipe $\rho$ from $E(H)$. If $\varphi^{-1}[\rho]$ contains one edge, say $u v \in E(G)$ with $\varphi(u)=\mu$ and $\varphi(v)=\nu$, then delete $u v$ from $E(G)$, insert two new vertices $u^{\prime}, v^{\prime}$ and new edges $u u^{\prime}, v v^{\prime}$ into $G$, and update 

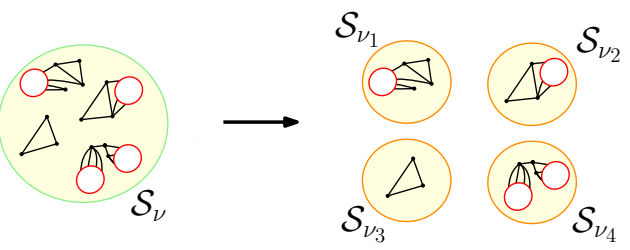

Figure 4: An atomic embedding of $G$ on $\mathcal{S}(\nu)$ and $\bigcup_{i} \mathcal{S}\left(\nu_{i}\right)$ before and after, respectively, operation Split $(\nu)$. The operation splits an atom $\nu \in V(H)$, for which $G_{\varphi}(\nu)$ is disconnected, into as many atoms as the number of connected components in $G_{\varphi}(\nu)$.

$\varphi$ with $\varphi\left(u^{\prime}\right)=\mu$ and $\varphi\left(v^{\prime}\right)=\nu$. If $\varphi^{-1}[\rho]$ contains two edges, say $u_{i} v_{i} \in E(G)$ with $\varphi\left(u_{i}\right)=\mu$ and $\varphi\left(v_{i}\right)=\nu$, for $i \in\{1,2\}$, then delete both $u_{1} v_{1}$ and $u_{2} v_{2}$ from $E(G)$, insert two new vertices $u^{\prime}, v^{\prime}$ and new edges $u_{1} u^{\prime}$, $u_{2} u^{\prime}, v_{1} v^{\prime}$, and $v_{2} v^{\prime}$ into $G$, and update $\varphi$ with $\varphi\left(u^{\prime}\right)=\mu$ and $\varphi\left(v^{\prime}\right)=\nu$.

Since the virtual vertices that correspond to $\rho$ in $G_{\varphi}(\mu)$ and $G_{\varphi}(\nu)$ have fixed rotations, by Observation 1, the following is straightforward.

Lemma 2.1. For every instance $\varphi: G \rightarrow H$ of atomic embeddability, and every pipe $\rho \in E(H)$, whose corresponding vertices in local graphs have degree less than 3 , operation Suppress $(\rho)$ produces an equivalent instance.

We define an operation that splits an atom $\nu$ if $G_{\varphi}(\nu)$ is disconnected, see Fig. 4

Split $(\nu)$. We are given a local graph $G_{\varphi}(\nu)$ whose connected components are $C_{1}, \ldots, C_{k}$, for some $k \in \mathbb{N}$. Delete $\nu$ from $H$, introduce new vertices $\nu_{1}, \ldots, \nu_{k}$ in $V(H)$, and introduce a pipe $\nu_{i} \mu$ for every $\rho=\nu \mu \in$ $E(H)$ such that $\rho$ corresponds to a virtual vertex of $C_{i}$. Finally, redefine $\varphi$ on $V\left(\varphi^{-1}[\nu]\right)$ as follows: Put $\varphi(v)=\nu_{i}$ if $v \in V\left(C_{i}\right)$.

By Observation 1. the following is straightforward.

Lemma 2.2. For every instance $\varphi: G \rightarrow H$ of atomic embeddability, an application of Split $(\nu)$ produces an equivalent instance.

Preprocessing $(\varphi)$. Input: an instance $\varphi: G \rightarrow H$ of atomic embeddability.

(1) For every pipe $\rho \in E(H)$ with $\left|\varphi^{-1}[\rho]\right| \leq 2$, apply Suppress $(\rho)$.

(2) For every atom $\nu \in V(H)$, where $G_{\varphi}(\nu)$ is disconnected, apply Split $(\nu)$.

Lemma 2.3. For an instance $\varphi: G \rightarrow H$ of atomic embeddability of size $n$, Preprocessing runs in $O(n)$ time and returns an equivalent normal instance $\varphi^{\prime}$.
Proof. By Lemmas 2.1 and 2.2, the instance $\varphi^{\prime}$ is equivalent to $\varphi$. Step (1) eliminates virtual vertices of degree less than 3, and Step (2) does not change the degree of any vertex in local graphs. Step (2) splits the local graphs $G_{\varphi}(\nu), \nu \in V(H)$, into connected components. Hence, $\varphi^{\prime}$ is normal. Step (1) runs in $O(1)$ time for each pipe of degree less than 3. Step (2) runs in $O(m)$ time for every local graph $G_{\varphi}(\nu)$ with $m=m(\nu)$ edges; which yields an overall running time of $O(n)$.

Data Structures. For a normal instance $\varphi: G \rightarrow$ $H$, let $\mathcal{G}$ be the disjoint union of all local graphs $G_{\varphi}(\nu)$, $\nu \in V(H)$. We maintain the graphs $G$ and $\mathcal{G}$ by adjacency lists. We maintain the set $V(H)$ of atoms implicitly: Each connected component in $\mathcal{G}$ corresponds to an atom $\nu \in V(H)$. We maintain the set $E(H)$ of pipes as follows: For every pipe $\rho \in E(H)$, we maintain two pointers to the two virtual vertices in $\mathcal{G}$ that corresponds to $\rho$; and also maintain the set $\varphi^{-1}[\rho] \subset E(G)$ of edges mapped to $\rho$ in a doubly linked list. Furthermore, for each edge $u v \in \varphi^{-1}[\rho]$, with $\varphi(u)=\mu$ and $\varphi(v)=\nu$, we maintain a pointer to $\rho$, and to the edge in $G_{\varphi}(\mu)$ (resp., $\left.G_{\varphi}(\nu)\right)$ that joins the virtual vertex corresponding to $\rho$ and $u$ (resp., $v$ ).

For every connected component $G_{\varphi}(\nu)$ of $\mathcal{G}$, we maintain $G_{\varphi}^{-}(\nu)$ (i.e., the multigraph obtained by supressing vertices of degree 2), if $G_{\varphi}(\nu)$ is not a cycle, by adjacency lists. Furthermore, we maintain the block tree of $G_{\varphi}^{-}(\nu)$, which is a bipartite graph that represents incidences between cut vertices and blocks (i.e., maximal 2-connected components). For each block of $G_{\varphi}^{-}(\nu)$, we also maintain an SPQR decomposition tree introduced by Di Battista and Tamassia [21, which is a hierarchical decomposition used for representing all 2 -cuts and their bridges. For each vertex $v$ of $\mathcal{G}$, we maintain indicator variables that record whether $v$ is an ordinary or virtual vertex, whether it is a proper cut vertex or contained in a proper 2-cut. At initialization, all these data structures can be computed in linear time in the size of $G$ and $H$. The data structures can be updated in linear time if necessary. (Currently available dynamic data structures for planarity testing and SPQR-trees, with sublinear update times, support some but not all of our graph operations.)

As we shall see, whenever our algorithm creates a pipe of degree less than 3 , it is immediately suppressed. If our algorithm modifies a graph $G_{\varphi}(\nu)$ in a way that it disconnects into components, then we assume that it immediately splits the corresponding atom $\nu$ as described above. In particular, our data structure supports the operation $\operatorname{Split}(\nu)$ in 0 time. In the remainder of the algorithm, we may assume that every instance of atomic embeddability is normal. 
2.4 Elementary Operations In this section we describe operations used in our algorithm for a given instance $\varphi: G \rightarrow H$ of atomic embeddibility. Each operation modifies the instance $\varphi$. Each operation is local in the sense that it affects an atom $\nu$ and possibly one or two of its neighbors. That is, the modifications incur changes in $G_{\varphi}(\nu)$, and possibly in $G_{\varphi}\left(\nu^{\prime}\right)$, for some of the neighbors $\nu^{\prime}$ of $\nu$.

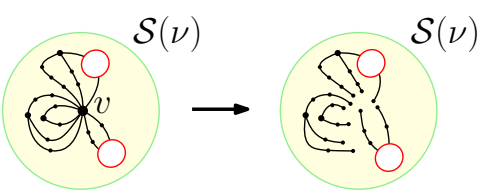

Figure 5: An atomic embedding of $G$ on $\mathcal{S}(\nu)$ before and after operation $\operatorname{Detach}(v)$. The operation turns an ordinary vertex $v$, the center of p-star $G_{\varphi}(\nu)$, into $\operatorname{deg}(v)$ leaves.

The following operation turns an ordinary vertex $v$ into $\operatorname{deg}(v)$ leaves, see Fig. 5 .

$\operatorname{Detach}(v)$. Let $v$ be an ordinary vertex in a graph $G_{\varphi}(\nu)$ such that every $\{v\}$-bridge is a p-path (that is, either $G_{\varphi}(\nu)$ is a p-star with center $v$, or $G_{\varphi}(\nu)$ is a ppath with a pole at $v)$. Let $v u_{1}, \ldots, v u_{\operatorname{deg}(v)}$ denote the edges incident to $v$ in $G$. Remove $v$ and its incident edges from $G$. Then introduce $\operatorname{deg}(v)$ new vertices $v_{1}, \ldots, v_{\operatorname{deg}(v)}$ and add edges $u_{i} v_{i}$, for all $i \in[\operatorname{deg}(v)]$ to $G$. Finally, define $\varphi\left(v_{i} u_{i}\right)=\varphi\left(v u_{i}\right)$.

By Observation 1, the following is straightforward.

Lemma 2.4. For an instance $\varphi: G \rightarrow H$ of atomic embeddability, Detach $(v)$ produces an equivalent instance $\varphi^{\prime}$. The operation can be implemented in $O(\operatorname{deg}(v))$ time.

In the following we define the operation of enclosing a bridge in $G_{\varphi}(\nu)$, see Fig. 6. This operation is analogous to stretching of a local branch in 14] except that we apply it in a more general setting.

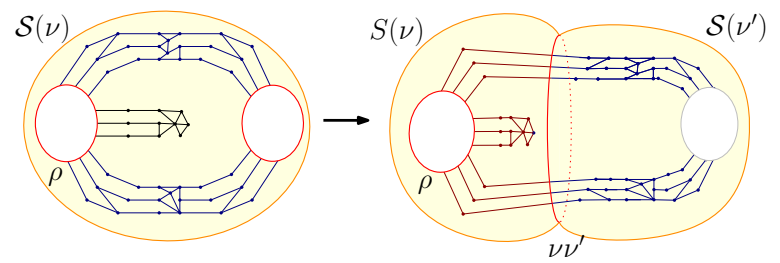

Figure 6: An atomic embedding of $G$ on $\mathcal{S}(\nu)$ before and after operation Enclose $(B)$, where $B$ (colored blue) is a $\{v\}$-bridge of $G_{\varphi}(\nu)$ and $v$ is a virtual vertex corresponding to the pipe $\rho$.
Enclose $(B)$. We are given a $\left\{v_{1}, \ldots, v_{k}\right\}$-bridge $B$ in $G_{\varphi}(\nu)$. The operation does not modify $G$ except for subdividing its edges. We first describe the changes in $H$, and then the changes in the local graphs. Create a new atom $\nu^{\prime}$ and a new pipe $\nu \nu^{\prime}$. Replace every pipe $\rho=\mu \nu$ that corresponds to a virtual vertex in $B \backslash\left\{v_{1}, \ldots, v_{k}\right\}$ with a new pipe $\mu \nu^{\prime}$. For every ordinary vertex $u \in V(B) \backslash\left\{v_{1}, \ldots, v_{k}\right\}$, set $\varphi(u)=\nu^{\prime}$. For every edge $e \in E(G)$, for which the pipe $\varphi(e)=\rho=\mu \nu$ has been replaced by $\rho^{\prime}=\mu \nu^{\prime}$, set $\varphi(e)=\rho^{\prime}$. If $v_{i} u \in E(B)$, and $v_{i}$ or $u$ is a virtual vertex of $G_{\varphi}(\nu)$, then subdivide $\overline{v_{i} u} \in E(G)$ by a vertex $w$ and define $\varphi(w)$ as follows: If $v_{i}$ is virtual, then put $\varphi(w)=\nu$; otherwise put $\varphi(w)=\nu^{\prime}$. Finally, update the definition of $\varphi$ on the edges of $B$ according to the value of $\varphi$ on the vertices of $G$ (this is uniquely determined since $\nu \nu^{\prime}$ is not a multiple pipe in $E(H)$ ).

For the purpose of the running time analysis the effect of the operation on $G_{\varphi}(\nu)$ is that we move the subgraph induced by $B \backslash\left\{v_{1}, \ldots, v_{k}\right\}$ from $G_{\varphi}(\nu)$ into a new graph $G_{\varphi}\left(\nu^{\prime}\right)$, and introduce a virtual vertex corresponding to the pipe $\nu \nu^{\prime}$ in both $G_{\varphi}(\nu)$ and $G_{\varphi}\left(\nu^{\prime}\right)$, whose degree is $\sum_{i=1}^{k} \operatorname{deg}_{B}\left(v_{i}\right)$. We will be often tacitly using the following lemma.

LEMma 2.5. Given an instance of atomic embeddability $\varphi$, an application of Enclose $(B)$ results in an equivalent instance $\varphi^{\prime}: G^{\prime} \rightarrow H^{\prime}$. The operation can be implemented in $O\left(\sum_{i=1}^{k} \operatorname{deg}_{B}\left(v_{i}\right)\right)$ time.
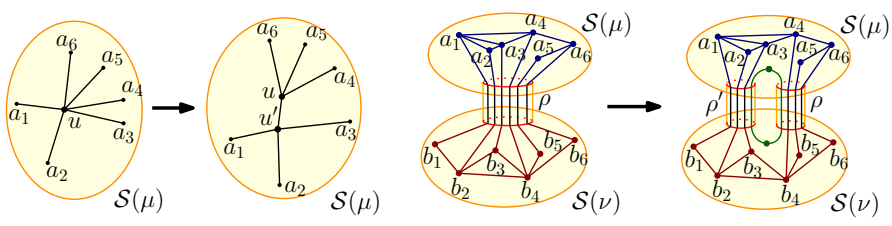

Figure 7: An atomic embedding of $G$ on $\mathcal{S}(\nu)$ before and after applying $\operatorname{Stretch}\left(u,\left\{u v_{1}, u v_{2}, u v_{3}\right\}\right)$. Vertex $u$ is either ordinary (left) or virtual (right). If $u$ is a virtual vertex, it corresponds to a pipe $\rho=\mu \nu$.

In the following we define the operation that replaces a vertex $u$ in $G_{\varphi}(\mu)$ by an edge $u u^{\prime}$, and distributes the edges incident to $u$ among $u$ and $u^{\prime}$. The operation produces an equivalent instance if the rotation of $u$ is fixed, see Fig. 7

$\operatorname{Stretch}\left(u, E_{u}\right)$. We are given a vertex $u$ in $G_{\varphi}(\mu)$ and a set $E_{u}=\left\{u a_{1}, \ldots, u a_{\ell}\right\}$ of edges incident to $u$ where $0<\left|E_{u}\right|<\operatorname{deg}(u)$. We distinguish between two cases, depending on whether $u$ is an ordinary or a virtual vertex. If $u$ is ordinary, then remove the edges $u a_{1}, \ldots, u a_{\ell}$, introduce a new vertex $u^{\prime}$ and a new edge $u u^{\prime}$, as well as new edges $u^{\prime} a_{1}, \ldots, u^{\prime} a_{\ell}$ in $G_{\varphi}(\mu)$. 
If $u$ is virtual, then let $\rho=\mu \nu \in E(H)$ be the pipe corresponding to the virtual vertex $u$ in $G_{\varphi}(\mu)$ and $v$ in $G_{\varphi}(\nu)$; and assume that $\overline{u a_{i}}=a_{i} b_{i}$, for $i \in[\ell]$, where $b_{1}, \ldots, b_{\ell}$ are vertices in $G_{\varphi}(\nu)$. Do the following: Introduce a new pipe $\rho^{\prime}=\mu \nu$ in $H$ corresponding to new virtual vertices $u^{\prime}$ in $G_{\varphi}(\mu)$ and $v^{\prime}$ in $G_{\varphi}(\nu)$; introduce a new connected component in $G$, which is a cycle with two vertices and two parallel edges $f, f^{\prime}$ forming a multiple edge such that $\varphi(f)=\rho$ and $\varphi\left(f^{\prime}\right)=\rho^{\prime}$; and finally, modify $\varphi$ by setting $\varphi\left(a_{i} b_{i}\right)=\rho^{\prime}$, for all $i \in[\ell]$. In local graphs this corresponds to replacing $u a_{i}$ and $v b_{i}$ with $u^{\prime} a_{i}$ and $v^{\prime} a_{i}$, respectively, for all $i \in[\ell]$; and inserting two new edges $u v$ and $u^{\prime} v^{\prime}$ in the two local graphs, respectively, and subdividing each with an ordinary vertex.

For the purposes of the running time analysis (below) the effect of the operation can be seen as the replacement of $u$ by an edge whose two endpoints have degrees $\ell+1$ and $\operatorname{deg}(u)-\ell+1$, respectively (hence the sum of their degrees equals $\operatorname{deg}(u)+2$ ). If $u$ is a virtual vertex (i.e., corresponds to a pipe between two atoms), then both virtual vertices corresponding to the same pipe go through these changes. By Observation 1 the following is straightforward.

Lemma 2.6. Given an instance of atomic embeddability $\varphi$ such that the edges in $E_{u}$ are incident to $u$, and are consecutive in the rotation of vertex $u$ in every embedding of $G_{\varphi}(\mu)$ inherited from an atomic embedding of $G$, then the operation $\operatorname{Stretch}\left(u, E_{u}\right)$ produces an equivalent instance.

Corollary 2.1. For an instance $\varphi: G \rightarrow H$ of atomic embeddability, if a vertex $u \in V\left(G_{\varphi}(\nu)\right)$ has a fixed rotation, in which the edges in $E_{u}$ are consecutive, then $\operatorname{Stretch}\left(u, E_{u}\right)$ produces an equivalent instance.

The operation of contraction that follows is applied to an edge $\rho=\nu \mu$ of $H$ and it produces an equivalent instance if each of $G_{\varphi}(\nu)$ and $G_{\varphi}(\mu)$ is a p-star or ppath.

Contract $(\rho)$. We are given a pipe $\rho=\mu \nu$ such that $\rho$ is the only pipe between $\mu$ and $\nu$. Contract the pipe $\mu \nu$ in $H$ into an atom $\langle\mu \nu\rangle$ and change $\varphi$ accordingly (that is, put $\varphi(u)=\langle\mu \nu\rangle$ for all the vertices mapped by $\varphi$ to $\mu$ or $\nu)$. Let $\varphi^{\prime}$ denote the resulting instance. Note that $G_{\varphi^{\prime}}(\langle\mu \nu\rangle)$ might be disconnected, in which case operation Split $(\langle\mu \nu\rangle)$ is automatically applied to obtain a normal instance, as explained in Section 2.3 . Since $\rho$ is the only pipe between $\mu$ and $\nu$ the operation does not introduce a loop in $H$.

Several incarnations of the following lemma, which is a consequence of Belyi's theorem [6], were proved in related papers; see for example, [2, Lemma 3.2], 26, Claim 7], or [27, Lemma 6].

Lemma 2.7. Let $\mu \nu \in E(H)$ be a pipe such that either (i) both $G_{\varphi}(\mu)$ and $G_{\varphi}(\nu)$ are p-stars, or (ii) $G_{\varphi}(\mu)$ or $G_{\varphi}(\nu)$ is a p-path; and in both cases, $\rho$ corresponds to vertices $u$ and $v$ of maximum degree in $G_{\varphi}(\mu)$ and $G_{\varphi}(\nu)$, respectively. Then Contract $(\mu \nu)$ produces an equivalent instance $\varphi^{\prime}$.

Proof. Denote by $\varphi^{\prime}: G \rightarrow H^{\prime}$ the map returned by Contract $(\mu \nu)$. First assume that $\varphi: G \rightarrow H$ is atomic embeddable. Then there exists an atomic embedding $\mathcal{E}: G \rightarrow \mathcal{H}$ (where every vertex $a \in V(G)$ is embedded in $\mathcal{S}(\varphi(a))$; and every edge $a b \in E(G)$ is embedded as a Jordan arc in $\mathcal{S}(\varphi(a)) \cup \mathcal{S}(\varphi(b))$ as specified in the definition of atomic embedding). Let $\mathcal{S}(\langle\mu \nu\rangle)=$ $\mathcal{S}(\mu) \cup \mathcal{S}(\nu)$. Then the thickening $\mathcal{H}^{\prime}$ of $H^{\prime}$ equals $\mathcal{H}$, and the embedding $\mathcal{E}: G \rightarrow \mathcal{H}=\mathcal{H}^{\prime}$ witnesses that $\varphi^{\prime}: G \rightarrow H^{\prime}$ is atomic embeddable.

Conversely, assume that $\varphi^{\prime}: G \rightarrow H^{\prime}$ is atomic embeddable. Let $\mathcal{E}^{\prime}: G \rightarrow \mathcal{H}^{\prime}$ be an atomic embedding. Consider the restriction of $\mathcal{E}^{\prime}: G \rightarrow \mathcal{H}^{\prime}$ on the surface $\mathcal{S}(\langle\mu \nu\rangle)$. Filling the holes of $\mathcal{S}(\langle\mu \nu\rangle)$ with discs, and then contract them to points, to obtain an embedding of $G_{\varphi^{\prime}}(\langle\mu \nu\rangle)$ on the sphere $S^{2}$.

First, assume that (i) both $G_{\varphi}(\mu)$ and $G_{\varphi}(\nu)$ are pstars: $G_{\varphi}(\mu)$ is the union of internally vertex disjoint paths between $u$ and a vertex set $V_{a}$, and similarly $G_{\varphi}(\nu)$ is the union of internally vertex disjoint paths between $v$ and a vertex set $V_{b}$. Consequently, $G_{\varphi^{\prime}}(\langle\mu \nu\rangle)$ is the union of internally vertex disjoint paths between vertices in $V_{a}$ and $V_{b}$. (Note that $G_{\varphi^{\prime}}(\langle\mu \nu\rangle)$ need not be connected.) By suppressing the internal vertices of the paths between $V_{a}$ and $V_{b}$, we obtain an embedding of a bipartite multigraph $G_{\varphi^{\prime}}^{-}(\langle\mu \nu\rangle)$ with partite sets $V_{a}$ and $V_{b}$ on $S^{2}$.

By Belyi's theorem [6], there exists a Jordan curve $\beta: S^{1} \rightarrow S^{2}$ that intersects every edge of $G_{\varphi^{\prime}}^{-}(\langle\mu \nu\rangle)$ in exactly one point, and the intersection is transversal. The curve $\beta$ partitions $S^{2}$ into two parts, $A$ and $B$. We can subdivide the edges of $G_{\varphi^{\prime}}^{-}(\langle\mu \nu\rangle)$ to obtain an embedding of $G_{\varphi^{\prime}}(\langle\mu \nu\rangle)$ on a sphere such that the curve $\beta$ crosses an edge $e \in E\left(G_{\varphi^{\prime}}(\langle\mu \nu\rangle)\right)$ if and only if $e \in \varphi^{-1}[\rho]$. Consequently, by contracting $A$ (resp., $B$ ) into a vertex $v$ (resp., $u$ ), we obtain an embedding of $G_{\varphi}(\nu)$ (resp., $G_{\varphi}(\mu)$ ) on a sphere, where the vertices $u$ and $v$ have opposite rotations. Observation 1 now implies that $\varphi: G \rightarrow H$ is atomic embeddable.

Next assume that (ii) $G_{\varphi}(\mu)$ or $G_{\varphi}(\nu)$ is a p-path: Without loss of generality, assume that $G_{\varphi}(\mu)$ is a ppath, with poles $u$ and $w$. Consequently, $G_{\varphi^{\prime}}(\langle\mu \nu\rangle)$ is a subdivision of $G_{\varphi}(\nu)$, obtained by subdividing the edges incident to $v$. In particular $G_{\varphi^{\prime}}^{-}(\langle\mu \nu\rangle)$ is isomorphic 


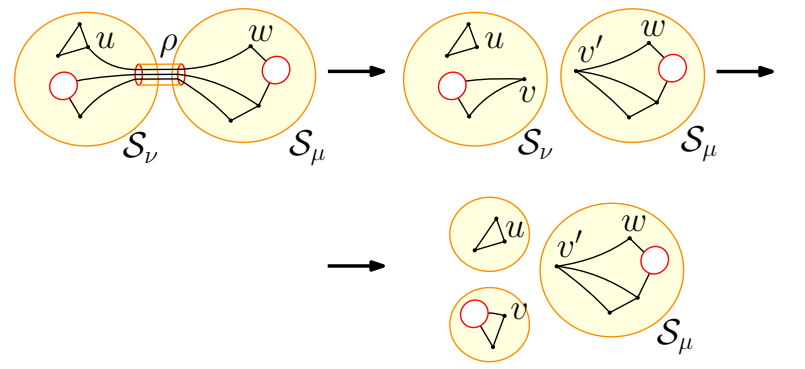

Figure 8: An atomic embedding of $G$ on $\mathcal{S}(\nu) \cup \mathcal{S}(\mu)$ before and after operation Delete $(u v)$, where $u v \in$ $G_{\varphi}(\nu)$ and $u w=\overline{u v}$. The operation reduces the degree of a virtual vertex $v$ of $G_{\varphi}(\nu)$ such that $v$ is incident to a cut-edge, and at most 3 edges of $G$ pass through its corresponding pipe $\rho$.

to $G_{\varphi}^{-}(\nu)$, where vertex $w$ in $G_{\varphi^{\prime}}^{-}(\langle\mu \nu\rangle)$ corresponds to vertex $v$ in $G_{\varphi}^{-}(\nu)$. By imposing the rotation of $w$ on $v$ (and the opposite rotation on $u$ ), Observation 1 implies that $\varphi: G \rightarrow H$ is atomic embeddable, completing the proof.

Our last operation deletes a cut edge of a subcubic local graph; see Fig. 8 for an illustration.

Delete $(e)$. We are given a cut edge $e=u v$ in a subcubic local graph $G_{\varphi}(\nu)$. If both $u$ and $v$ are ordinary vertices, then delete $u v$ from $E(G)$ (thereby disconnecting $G_{\varphi}(\nu)$ into two components and invoking Split $(\nu))$. Else assume w.l.o.g. that $u$ is ordinary and $v$ is virtual. Let $\rho=\mu \nu$ be the pipe that corresponds to $v$ in $G_{\varphi}(\nu)$ and a vertex $v^{\prime}$ in $G_{\varphi}(\mu)$, and let $u w \in E(G)$ be the edge corresponding to $u v$, that is, $u w=\overline{u v}=\overline{v^{\prime} w}$, where $v^{\prime} w \in G_{\varphi}(\mu)$. Delete the edge $u w$ from $G$ (thereby reducing the degree of $\rho$ to 2), then Suppress $(\rho)$ (which turns $v$ and $v^{\prime}$ into ordinary vertices), and finally insert an edge $v^{\prime} w$ into both $G$ and $G_{\varphi}(\mu)$.

Lemma 2.8. For every instance $\varphi: G \rightarrow H$ of atomic embeddability, an application of Delete(e) produces an equivalent instance $\varphi^{\prime}$.

2.5 Toroidal Instance An instance $\varphi: G \rightarrow H$ is toroidal if $H$ is a cycle and for every atom $\nu \in$ $V(H)$, the graph $G_{\varphi}(\nu)$ is a p-path in which both poles are virtual vertices, and correspond to the two pipes incident to $\nu$.

Given an instance $\varphi: G \rightarrow H$ and a subgraph $H^{\prime} \subseteq H$, such that the restriction of $\varphi$ to $G^{\prime}=\varphi^{-1}\left[H^{\prime}\right]$, denoted $\varphi^{\prime}: G^{\prime} \rightarrow H^{\prime}$, is toroidal, we say that $H^{\prime}$ is a toroidal cycle in $H$.

In this section, we show how to decide toroidal instances of atomic embeddability in linear time. First, note that in a toroidal instance every ordinary vertex has degree 2 , hence $G$ is a disjoint union of cycles, say $C_{1}, \ldots, C_{t}$, for some $t \in \mathbb{N}$. Furthermore, $\varphi$ maps each cycle $C_{k}, k \in[t]$, to a walk that winds around $H$ once or more times.

Lemma 2.9. Let $\varphi: G \rightarrow H$ be a toroidal instance of atomic embeddability, where $H$ is a cycle, and $G$ is a vertex disjoint union of cycles $C_{1}, \ldots, C_{t}$. The instance $\varphi$ is positive if and only if $\varphi\left(C_{k}\right)$ is a walk of the same length for all $k \in[t]$ (that is, every cycle winds around the torus $\mathcal{H}$ the same number of times).

Roughly speaking, Lemma 2.9 follows by the intersection form of the closed curves on the torus. Indeed, whether a pair of curves could be crossing free on the torus is governed by their homology classes 30, Example 2A.2. and Corollary 3A.6.(b)] over $\mathbb{Z}$.

Corollary 2.2. We can decide whether a toroidal instance $\varphi: G \rightarrow H$ is atomic embeddable in time $O(n)$, where $n$ is the number of edges and vertices in $G$.

2.6 The Subcubic Case An instance $\varphi: G \rightarrow H$ of ATOMIC EMBEDDABILITY is subcubic if $G_{\varphi}(\nu)$ is subcubic (i.e., its maximum degree is at most 3 ) for every $\nu \in V(H)$. In this section, we show how to decide subcubic instances of ATOMIC EMBEDDIBILITY in linear time. By Observation 11 it is enough to check whether all graphs $G_{\varphi}(\nu), \nu \in V(H)$, are planar, and they each have embeddings in the plane such that for every pipe $\mu \nu \in E(H)$, the virtual vertices corresponding to $\mu \nu$ in $G_{\varphi}(\mu)$ and $G_{\varphi}(\nu)$ have opposite rotations.

Planarity testing for a graph takes linear time 31 . Let $n$ be the number of vertices and edges in $G$. Then the disjoint union of all local graphs $\mathcal{G}$ has $O(n)$ size (since each vertex in $V(G)$ corresponds to a unique ordinary vertex, and every edge in $E(G)$ corresponds to one or two edges in $\mathcal{G}$ ). Hence planarity testing for $\mathcal{G}$ takes $O(n)$ time.

In the subcubic case, every vertex in the local graphs $G_{\varphi}(\nu), \nu \in V(H)$, has at most two possible rotations (including the vertices of 1 - and 2 -cuts). We show how to encode the possible embeddings of each local graph by a boolean variable, and then reduce the existence of compatible embeddings to a 2SAT formula which can be solved in $O(n)$ time.

We start with a postprocessing algorithm that eliminates 1- and 2-edge-cuts from local graphs.

Postprocessing. We are given a subcubic instance $\varphi: G \rightarrow H$ of atomic embeddability.

(1) While there exists a cut edge $e$ in some $G_{\varphi}(\nu)$, $\nu \in V(H)$, apply Delete $(e)$ 
(2) While there exists a proper 2-edge-cut $\{e, f\}$ in a local graph of $\varphi$, such that $e=u_{1} v_{1}$ and $f=u_{2} v_{2}$, where both $v_{1}$ and $v_{2}$ are in a $\left\{u_{1}, u_{2}\right\}$-bridge $B$ of $G_{\varphi}(\nu)$, apply Enclose $(B)$ (creating a new pipe $\rho_{B}$ of degree 2), and Suppress $\left(\rho_{B}\right)$.

LEMma 2.10. We can decide whether a subcubic instance $\varphi: G \rightarrow H$ is atomic embeddable in $O(n)$ time, where $n$ is the number of edges and vertices in $G$ and $H$.

2.7 Main Algorithm We define two subroutines and then present our main algorithm. Subroutine 1 ensures that our instance has some desirable properties, and Subroutine 2 decreases the maximum degree over all local graphs $G_{\varphi}(\nu)$, for all atoms $\nu \in V(H)$, that are not contained in a toroidal cycle $C$ of $H$.

The crucial part of our algorithm reduces the maximum degree in local graphs over all atoms that are not in toroidal cycles. Specifically, for an instance $\varphi$ of atomic embeddability, let

- $V^{*}(H)$ (resp., $E^{*}(H)$ ) be the set of atoms (resp., pipes) in $H$ that are not in any toroidal cycle of $H$; and

- let $\Delta(\varphi)$ be the maximum degree over all vertices of all local graphs $G_{\varphi}(\nu), \nu \in V^{*}(H)$, if $V^{*}(H) \neq \emptyset$, and let $\Delta(\varphi)=2$ if $V(H)=\emptyset$.

We first call Subroutine 1 for a normal instance $\varphi$, and show that it returns an equivalent instance in which the proper 1- and 2-cuts in local graphs $G_{\varphi}(\nu)$, $\nu \in V^{*}(H)$, are in a special form, as described in terms of the following definition.

DeFINITION 2. An instance $\varphi$ of atomic embeddability is $d$-nice, for $d \geq 3$, if it meets the following two conditions:

(N1) $\Delta(\varphi) \leq d$.

(N2) If $\operatorname{deg}(v)=d$ for a vertex $v$ of some local graph $G_{\varphi}(\nu), \nu \in V(H)$, then $v$ has a fixed rotation, or $G_{\varphi}(\nu)$ is a p-path or a p-star.

(N3) If $\rho=\mu \nu \in E(H)$, such that both $G_{\varphi}(\mu)$ and $G_{\varphi}(\nu)$ are $p$-stars, and $\rho$ corresponds to virtual vertices of degree at least $d$, then $\rho$ is the only pipe between $\mu$ and $\nu$.

We present a subroutine that takes a normal instance $\varphi$ as an input, and returns a $\Delta$-nice normal instance for the maximum degree $\Delta$ over all local graphs, that is, $\Delta=\Delta(\varphi)$ (as shown in Lemma 2.11 and Corollary 2.4 below).

Subroutine 1. Input: a normal instance $\varphi$ of atomic embeddability, where $\Delta(\varphi) \geq 4$.
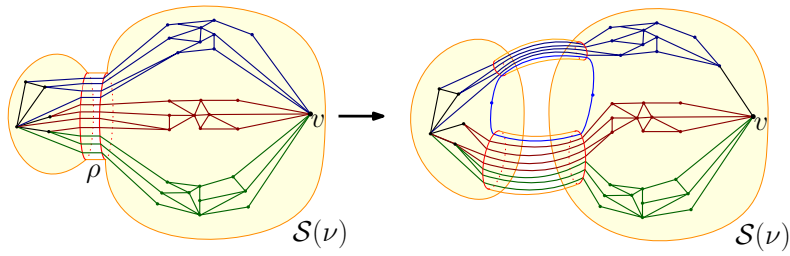

Figure 9: An atomic embedding of $G$ on $\mathcal{S}(\nu)$ before and after Step (i), where $u$ is a virtual vertex of $G_{\varphi}(\nu)$ corresponding to the pipe $\rho$, and $v$ is an ordinary vertex of $G_{\varphi}(\nu)$.

(i) While there is a proper 2-cut $\{u, v\}$ and a nonseparable $\{u, v\}$-bridge $B$ in $G_{\varphi}(\nu)$, for some $\nu \in V(H)$, such that $\max \{\operatorname{deg}(u), \operatorname{deg}(v)\}=\Delta$, but neither $u$ nor $v$ is a cut vertex, then do the following: Perform $\operatorname{Stretch}\left(u, E_{u}\right)$, where $E_{u}$ is the set of edges in $E(B)$ incident to $u$; and perform $\operatorname{Stretch}\left(v, E_{v}\right)$, where $E_{v}$ is the set of edges in $E(B)$ incident to $v$. If $u$ or $v$ is a virtual vertex corresponding to a pipe $\mu \nu$ and $G_{\varphi}(\mu)$ is nonplanar, report that the instance $\varphi$ is not atomic embeddable and exit the subroutine.

(ii) While there is a proper 2-edge-cut $\{e, f\}$ in $G_{\varphi}(\nu)$, for some $\nu \in V(H)$, then let $e=u_{1} v_{1}$ and $f=u_{2} v_{2}$ such that both $v_{1}$ and $v_{2}$ are in a $\left\{u_{1}, u_{2}\right\}$-bridge $B$ of $G_{\varphi}(\nu)$, then apply Enclose $(B)$ (creating a new pipe $\rho_{B}$ of degree 2$)$, and Suppress $\left(\rho_{B}\right)$.

(iii) While there is a proper cut vertex $v$ with $\operatorname{deg}(v)=$ $\Delta$ in some local graph of $\varphi$, then successively apply Enclose $(B)$ for every bridge $B$ of $v$ (thereby turning every bridge of $v$ into a p-path). Apply $\operatorname{Suppress}\left(\rho_{B}\right)$ if applicable.

In Section 2.8 (cf. Corollary 2.4), we show that Subroutine 1 terminates and analyse its running time. Here we prove that if it terminates, it returns a $\Delta(\varphi)$ nice instance.

LEMMA 2.11. For an instance $\varphi$ of atomic embeddibility, if Subroutine 1 terminates, it either returns an equivalent, normal, and $\Delta(\varphi)$-nice instance $\varphi^{\prime}$, or reports that $\varphi$ is not atomic embeddable.

Proof. Let $\Delta=\Delta(\varphi)$ for short. By Lemmas 2.1 2.5. and 2.6. Subroutine 1 returns an equivalent instance $\varphi^{\prime}$ upon termination. Note that instance $\varphi^{\prime}$ is normal, since we apply Suppress $\left(\nu_{B}\right)$ to any pipe of degree less than 3. The operations in Subroutine 1 do not increase the maximum degree in any local graph outside of toroidal cycles; and make no changes at all in local graphs in toroidal cycles. Consequently, $\Delta\left(\varphi^{\prime}\right) \leq \Delta$. 


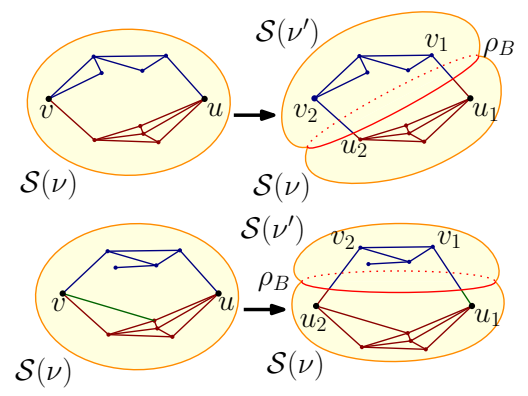

Figure 10: The operation Enclose $(B)$ in Step (ii) eliminates every proper 2-cut $\{u, v\}$ in $G_{\varphi}(\nu)$ that has exactly two $\{u, v\}$-bridges. Either one of the $\{u, v\}$ bridges is separable (left), or there exists a separable $\left\{u_{1}, u_{2}\right\}$-bridge such that $\operatorname{deg}_{B}\left(u_{1}\right)=\operatorname{deg}_{B}\left(u_{2}\right)=1$ (right).

At the end of Step (i), every $\{u, v\}$-bridge is separable for every proper $\{u, v\}$-cut where $\max \{\operatorname{deg}(u), \operatorname{deg}(v)\}=\Delta$; see Fig. 9 . We consider Step [ii) now. Suppose that $\{u, v\}$ is a proper 2cut in $G_{\varphi}(\nu)$, such that $\min \{\operatorname{deg}(u), \operatorname{deg}(v)\} \geq 3$ and $\max \{\operatorname{deg}(u), \operatorname{deg}(v)\}=\Delta$

If there exist exactly two (separable) $\{u, v\}$-bridges in $G_{\varphi}(\nu)$ (none of which is a subdivided edge as otherwise $\{u, v\}$ would not be a proper 2-cut), then Step(ii) eliminates the proper 2-cut $\{u, v\}$ by a single application of Enclose(.), due to Observation 2, and does not introduce any new proper 2-cut. Indeed, up to symmetry there are two cases to consider depending on whether $\operatorname{deg}_{B}(u)=1$ or $\operatorname{deg}_{B}(u)=\operatorname{deg}(u)-1$, and $\operatorname{deg}_{B}(v)=1$ or $\operatorname{deg}_{B}(v)=\operatorname{deg}(v)-1$; see Fig. 10 .

If there exist at least three (separable) $\{u, v\}$ bridges in $G_{\varphi}(\nu)$ such that $\max \{\operatorname{deg}(u), \operatorname{deg}(v)\}=\Delta$, then Step (ii) turns $G_{\varphi}(\nu)$ into a p-path with the poles $u$ and $v$.

Hence, at the end of Step (ii) for every proper 2-cut $\{u, v\}$ we have (a) $\max \{\operatorname{deg}(u), \operatorname{deg}(v)\}<\Delta$; or (b) $u$ or $v$ is a cut vertex of degree $\Delta$; or (c) $u$ and $v$ are the poles of a p-path. In particular, every vertex $w$ with $\operatorname{deg}(w)=\Delta$ in a local graph $G_{\varphi}(\mu)$, is a proper 1-cut, or a pole of a p-path, or has fixed rotation.

Step (iii) successively turns every cut vertex of degree $\Delta$ into the center of a p-star. It creates new 2 cuts within these p-stars and possibly in adjacent atoms, but it does not create any new vertex of degree $\Delta$. Hence, at the end of Subroutine $1 \varphi$ satisfies (N1) and (N2). For property (N3) note that by enclosing all the bridges of the center of every p-star in $\mathcal{G}$ of degree $\Delta$, Step (iii) eliminates possible problematic multiple pipes $\rho=\mu \nu$ in $H$, where $\rho$ corresponds to a pair of centers of p-stars $G_{\varphi}(\mu)$ and $G_{\varphi}(\nu)$. Overall, the instance $\varphi^{\prime}$
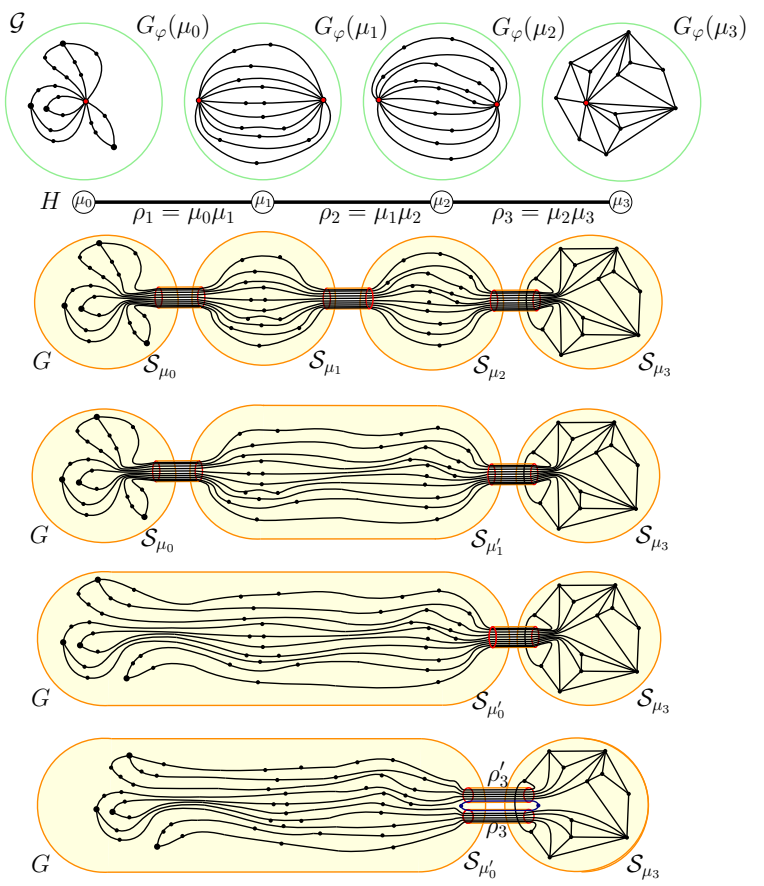

Figure 11: A path $\left(\rho_{1}, \rho_{2}, \rho_{3}\right)$ in $H$ through the atoms $\mu_{0}, \ldots, \mu_{3}$. The graph $G_{\varphi}\left(\mu_{0}\right)$ is a p-star, $G_{\varphi}\left(\mu_{1}\right)$ and $G_{\varphi}\left(\mu_{2}\right)$ are p-paths, and $G_{\varphi}\left(\mu_{3}\right)$ is 3-connected. In each local graph, the virtual vertices corresponding to $\rho_{1}, \rho_{2}$, or $\rho_{3}$ are vertices of maximum degree. The bottom three subfigures show the effect of Step (iv.a) and Step (v.c) of Subroutine 2 on the graph $G$ in this instance.

returned by Subroutine 1 upon termination meets both conditions (N1) (N3), consequently $\varphi^{\prime}$ is $\Delta$-nice.

Degree reduction. We are now ready to present the crucial subroutine of our algorithm that reduces $\Delta(\varphi)$ by eliminating all vertices of degree $\Delta(\varphi)$ that are not in toroidal cycles. (See Figs. 11 and 12 for the possible relations between virtual vertices of degree $\Delta(\varphi)$.)

Subroutine 2. Input: a normal and $\Delta$-nice instance $\varphi$ of atomic embeddability, where $\Delta=$ $\Delta(\varphi)$ and $\Delta \geq 4$.
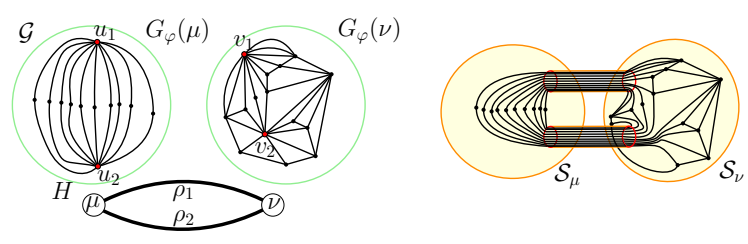

Figure 12: An illustration of the setting in Step (iv.b). analogous to Fig. 11. 
(iv) While there exists a pipe $\mu \nu \in E^{*}(H)$ of degree $\Delta$ such that $G_{\varphi}(\mu)$ or $G_{\varphi}(\nu)$ is a p-path, do the following. Suppose w.l.o.g. that $G_{\varphi}(\mu)$ is a p-path.

(a) If $\mu \nu$ is not a multiple edge apply Contract $(\mu \nu)$.

(b) Else there exists a pair of pipes $\rho_{1}$ and $\rho_{2}$ joining $\mu$ with $\nu$. Let $u_{i}$ and $v_{i}$, resp., be virtual vertices in $G_{\varphi}(\mu)$ and $G_{\varphi}(\nu)$ corresponding to $\rho_{i}$ for $i \in\{1,2\}$. Note that $u_{1}$ and $u_{2}$ are the poles of the p-path $G_{\varphi}(\mu)$; and both $v_{1}$ and $v_{2}$ are fixed due to (N2), as $\mu$ is not in a toroidal cycle. Apply $\operatorname{Stretch}\left(v_{1}, E_{1}\right)$ and $\operatorname{Stretch}\left(v_{2}, E_{2}\right)$, where $E_{i}$ is a set of $\lfloor\Delta / 2\rfloor$ consecutive edges in the rotation at $v_{i}$, for $i \in\{1,2\}$. If $G_{\varphi}(\mu)$ becomes nonplanar, report that the instance is not atomic embeddable and exit the subroutine.

(v) For every pipe $\mu \nu \in E^{*}(H)$ of degree $\Delta$ that corresponds to virtual vertices $u$ and $v$ in $G_{\varphi}(\mu)$ and $G_{\varphi}(\nu)$, respectively, do:

(a) If both $u$ and $v$ have fixed rotations (in $G_{\varphi}(\mu)$ and $G_{\varphi}(\nu)$, resp.), then check whether the two rotations are compatible. If they are incompatible, then report that $\varphi$ is not atomic embeddable and exit the subroutine. Otherwise apply $\operatorname{Stretch}\left(u, E_{u}\right)$, where $E_{u}$ is a set of $\lfloor\Delta / 2\rfloor$ consecutive edges in the rotation of $u$.

(b) If neither $u$ nor $v$ has a fixed rotation, then apply Contract $(\mu \nu)$. This contracts $\mu \nu$ into a new atom, denoted by $\langle\mu \nu\rangle$, and combines $G_{\varphi}(\mu)$ and $G_{\varphi}(\nu)$ into a new graph $G_{\varphi}(\langle\mu \nu\rangle)$. If $G_{\varphi}(\langle\mu \nu\rangle)$ is nonplanar, report that $\varphi$ is not atomic embeddable and exit the subroutine.

(c) Else assume w.l.o.g. that $u$ has fixed rotation in $G_{\varphi}(\mu)$, and is incident to edges $\left(u v_{1}, \ldots, u v_{\Delta}\right)$ in this cyclic rotation order. Successively apply $\operatorname{Stretch}(u,$.$) , turning ver-$ tex $u$ into an induced binary tree with $\Delta-2$ vertices. If $G_{\varphi}(\nu)$ is nonplanar, report that the instance is not atomic embeddable and exit the subroutine.

(vi) For every ordinary vertex $v \in V\left(G_{\varphi}(\nu)\right), \nu \in$ $V(H)$, with $\operatorname{deg}(v)=\Delta$ that has fixed rotation, apply $\operatorname{Stretch}\left(v, E_{v}\right)$, where $E_{v}$ is a set of $\lfloor\Delta / 2\rfloor$ consecutive edges in the rotation of $v$.

(vii) For every ordinary vertex $v \in V\left(G_{\varphi}(\nu)\right), \nu \in$ $V(H)$, with $\operatorname{deg}(v)=\Delta$ that is part of a 1 - or 2-cut, apply $\operatorname{Detach}(v)$.
This completes the description of Subroutine 2. In Section 2.8 we show that Subroutine 2 terminates and analyse its running time. In Lemma 2.13 below, we prove that if it terminates, it returns an instance $\varphi^{\prime}$ with $\Delta\left(\varphi^{\prime}\right)<\Delta(\varphi)$. We first clarify when an operation Stretch(.) can create a proper 1- or 2-cut.

Lemma 2.12. Let $u$ be a vertex in $G_{\varphi}(\nu)$ such that $\operatorname{deg}(u) \geq 4$, and assume that operation Stretch $(u,$. produces an instance $\varphi^{\prime}$ in which $u$ is replaced by an edge $u u^{\prime}$. If $u$ is not a proper 1-cut in $G_{\varphi}(\nu)$, then neither $u$ nor $u^{\prime}$ is a proper 1-cut in $G_{\varphi^{\prime}}(\nu)$. If u neither is a proper 1-cut nor belongs to a proper 2-cut in $G_{\varphi}(\nu)$, then neither $u$ nor $u^{\prime}$ belongs to a proper 2-cut in $G_{\varphi^{\prime}}(\nu)$.

Lemma 2.13. For a $\Delta(\varphi)$-nice instance $\varphi: G \rightarrow H$ of atomic embeddability, if Subroutine 2 terminates, then it either returns an equivalent normal instance $\varphi^{\prime}: G^{\prime} \rightarrow H^{\prime}$ such that $\Delta\left(\varphi^{\prime}\right)<\Delta(\varphi)$, or reports that $\varphi$ is not atomic embeddable.

Proof. Let $\Delta=\Delta(\varphi)$. We show that every step of Subroutine 2 maintains a $\Delta$-nice normal instance equivalent to $\varphi$ until it terminates; and it either returns such an instance $\varphi^{\prime}$ or reports that $\varphi^{\prime}$ is not atomic embeddable. Subroutine 2 maintains a normal instance until termination, since it does not create virtual vertices of degree 2 and Split(.) is applied automatically whenever a local graph disconnects into two or more components.

Step (iv.a) produces an equivalent instance by Lemma 2.7 Each Contract(.) operation merges a local graph $G_{\varphi}(\nu)$ with a p-path $G_{\varphi}(\mu)$ and produces a new local graph $G_{\varphi}(\langle\mu \nu\rangle)$, where $G_{\varphi}^{-}(\langle\mu \nu\rangle)$ is isomorphic to $G_{\varphi}^{-}(\nu)$, so the instance remains $\Delta$-nice. In Step (iv.b) the two invocation of Stretch(.) produce an equivalent instance by Lemma 2.6. At the end of Step (iv) none of the local graphs outside of toroidal cycles is a p-path of degree $\Delta$.

In Step (v.a), the rotation of the virtual vertices $u$ and $v$ must be compatible in any atomic embedding by Observation 1. If they are compatible, then operation Stretch $(u,$.$) produces an equivalent instance by Corol-$ lary 2.1: and the resulting instance is still $\Delta$-nice since no new proper 1-cut or 2-cut is introduced in $G_{\varphi}(\mu)$ and $G_{\varphi}(\nu)$ by Lemma 2.12

In Step (v.b) both $G_{\varphi}(\mu)$ and $G_{\varphi}(\nu)$ are p-star, centered at $u$, and $v$ resp., since instance is $\Delta$-nice, and p-paths of degree $\Delta$ have already been eliminated. By Lemma 2.7. Contract $(\mu \nu)$ produces an equivalent instance. The resulting instance is still $\Delta$-nice, since the maximum degree of $G_{\varphi}(\langle\mu \nu\rangle)$ is less than $\Delta$; and $G_{\varphi}(\langle\mu \nu\rangle)$ is planar if $\varphi$ is atomic embeddable by Observation 1

In Step (v.c) operation $\operatorname{Stretch}(u,$.$) for a fixed$ vertex $u$ yields an equivalent instance by Corollary 2.1 . 
If the rotation of $u$ is incompatible with a p-star centered at $v$, $\operatorname{Stretch}(u,$.$) may turn G_{\varphi}(\nu)$ into a nonplanar graph, and then $\varphi$ is not atomic embeddable by Observation 1. Note that $G_{\varphi}(\nu)$ is a p-star, since p-paths have been eliminated in Step (iv). Successive Stretch(.) operations eliminate the only vertex of degree $\Delta$ of $G_{\varphi}(\nu)$, namely $v$. The graph $G_{\varphi}(\mu)^{-}$remains 3connected by Lemma 2.12, and so the resulting instance is $\Delta$-nice.

The equivalence of Step (vi) follows by Lemma 2.6 and the resulting instance is still $\Delta$-nice, since operation $\operatorname{Stretch}(v,$.$) does not introduce a 1$-cut in $G_{\varphi}(\nu)$ that would violate the $\Delta$-nice property by Lemma 2.12 .

In Step (vii) operation $\operatorname{Detach}(u)$ is applied in a $\Delta$ nice instance, hence it produces an equivalent instance by Lemma 2.4, which obviously remains $\Delta$-nice.

For the remainder of the proof, assume that Subroutine 2 returns an instance $\varphi^{\prime}$. The operations in Subroutine 2 do not increase the maximum degree outside of toroidal cycles; and make no changes within toroidal cycles. Consequently, $\Delta\left(\varphi^{\prime}\right) \leq \Delta$.

It remains to prove that $\Delta\left(\varphi^{\prime}\right)<\Delta$, i.e., that Subroutine 2 eliminates vertices of degree $\Delta$ from all local graphs outside of toroidal cycles; call these vertices $\Delta$-critical. Since $\varphi$ is $\Delta$-nice, every $\Delta$-critical vertex in a local graph has a fixed rotation, or it is a center of a p-star, or a pole of a p-path. Steps (iv) (vii) each eliminate one or two $\Delta$-critical vertices (possibly a pair of corresponding virtual vertices), and do not create any new $\Delta$-critical vertices. Steps (iv) (v) eliminate all possible $\Delta$-critical virtual vertices; and Steps (vi) (vii) eliminate all $\Delta$-critical ordinary vertices. Since Subroutine 2 maintains a $\Delta$-nice instance, it ultimately eliminates all $\Delta$-critical vertices, and so $\Delta\left(\varphi^{\prime}\right)<\Delta$, as claimed.

Algorithm. We are given a normal instance $\varphi$ of atomic embeddability.

(I) While $\Delta(\varphi) \geq 4$, do the following.

(a) Call Subroutine 1 (which turns $\varphi$ into a $\Delta$-nice instance) followed by Subroutine 2 (which reduces $\Delta(\varphi)$ ). If Subroutine 1 or Subroutine 2 reports that the instance $\varphi$ is not atomic embeddable return False and terminate the algorithm.

(II) For each connected component $C$ of $H$, let $G(C)=$ $\varphi^{-1}[C]$.

(a) If $C$ is a toroidal cycle of $H$, decide atomic embeddability for $\left.\varphi\right|_{G(C)}$ using Corollary 2.2

(b) Else decide atomic embeddability for $\left.\varphi\right|_{G(C)}$ using Lemma 2.10 .
(III) If $\left.\varphi\right|_{G(C)}$ is atomic embeddable for all components $C$ of $H$, then return True; else return False.

In Section 2.8 we show that the Algorithm terminates and analyse its running time. Here we show that if it terminates it correctly decides the atomic embeddability problem.

Lemma 2.14. Suppose that Algorithm terminates for an instance $\varphi: G \rightarrow H$. Then the algorithm returns True if and only if $\varphi$ is atomic embeddable.

Proof. Since the input $\varphi$ is normal, it is a valid input for Subroutine 1 in the first iteration of Step (I.a). By Lemma 2.11. Subroutine 1 returns a $\Delta(\varphi)$-nice instance and therefore the input for Subroutine 2 is valid. In any subsequent iteration of Step (I.a) Subroutine 1 receives a valid input as Subroutine 2 returns a normal instance $\varphi^{*}$ (however, this instance need not be $\Delta\left(\varphi^{*}\right)$-nice). By Lemma 2.11 and Lemma 2.13, the while loop in Step(I) terminates after at most $\Delta(\varphi)-3$ iterations, and returns an equivalent instance (or correctly reports that $\varphi$ is not atomic embeddable). If the Algorithm proceeds to Steps (II) (III) the correctness of the output follows from Corollary 2.2 and Lemma 2.10

\subsection{Running Time Analysis}

Potential Functions. We measure the progress of the algorithm, for an instance $\varphi: G \rightarrow H$, using three parameters defined as follows. Recall that $\mathcal{G}$ denotes the disjoint union of all local graphs $G_{\varphi}(\nu), \nu \in V(H)$.

- Let $N(\varphi)=|V(\mathcal{G})|$, that is, the number of vertices of $\mathcal{G}$.

- let $N_{\geq 3}(\varphi)=\mid\{v \in V(\mathcal{G})$ : $\operatorname{deg}(v) \geq 3\} \mid$, i.e., the number of vertices of $\mathcal{G}$ of degree 3 or higher.

- Let the potential of $\varphi$ be

$$
\Phi(\varphi)=\sum_{v \in V(\mathcal{G})}(\max \{0, \operatorname{deg}(v)-\xi(v)\})^{\sigma(v)}
$$

where $\xi(v)=2$ and $\sigma(v)=3$ if $v$ is a proper cut vertex, $\xi(v)=2$ and $\sigma(v)=2$ if $v$ is part of a proper 2-cut but not a cut vertex, and $\xi(v)=3$ and $\sigma(v)=1$ otherwise.

Note that $\max \{0, \operatorname{deg}(v)-2\}=0$ if $\operatorname{deg}(v) \leq 2$, that is, the vertices of local graphs of degree less than 3 do not contribute to the potential. Clearly $\operatorname{deg}(v)<N(\varphi)$ for every $v \in V(\mathcal{G})$, and so $\Phi(\varphi) \leq N^{4}(\varphi)$ is a trivial upper bound. Our analysis hinges on the following charging scheme: 
Overview. We show below (Lemma 2.18) that each iteration of Step (I.a) of the Algorithm decreases the potential. This readily implies that the while loop in Step (I) terminates (hence the Algorithm terminates, which completes the proof of correctness). Recall that Step (I.a) runs Subroutines 1 and 2, that is, it applies Steps (i) (vii). We show that both the number of elementary operations performed and the number of new vertices created in Steps (i) (vii) are bounded from above by a constant times the decrease of the potential. Step (ii) does not change the potential, so we need additional machinery to bound its running time: We use the parameters $N(\varphi)$ and $N_{\geq 3}(\varphi)$. We continue with the specifics.

Analysis. Recall that each iteration of the while loop of Step (I) of the Algorithm, which calls Subroutine 1 followed by Subroutine 2. The two subroutine jointly perform Steps (i) (vii). We use the following notation. Assume that $\varphi_{0}$ is the input of Subroutine 1 , and we obtain instances $\varphi_{1}, \ldots, \varphi_{7}$ at the end of Step (i).... (vii) Denote by $\mathcal{G}_{i}$ the union of all local graphs in the instance $\varphi_{i}$ for $i=1, \ldots, 7$. The following lemma is helpful for the analysis of Step (i).

Lemma 2.15. Let $\{u, v\}$ be a proper 2-cut in a local graph $G_{\varphi}(\nu)$ such that $\max \{\operatorname{deg}(u), \operatorname{deg}(v)\} \geq 4$; and let $B$ be a nonseparable $\{u, v\}$-bridge. Then one iteration of the while loop in Step (i) produces an instance $\varphi^{\prime}$ such that $\Phi\left(\varphi^{\prime}\right)<\Phi(\varphi)$ and $N\left(\varphi^{\prime}\right) \leq N(\varphi)+4$.

COROLlary 2.3. Let $\varphi_{0}$ be a normal instance of atomic embeddability and let $\Delta=\Delta\left(\varphi_{0}\right)$. Then the while loop in Step (i) terminates and returns an instance $\varphi_{1}$, after at most $\Phi\left(\varphi_{0}\right)-\Phi\left(\varphi_{1}\right)$ iterations, such that $N\left(\varphi_{1}\right) \leq N\left(\varphi_{0}\right)+12\left(\Phi\left(\varphi_{0}\right)-\Phi\left(\varphi_{1}\right)\right)$.

Proof. By Lemma 2.15, each iteration of the while loop in Step (i) decreases the potential. Consequently, the while loop terminates, and performs at most $\Phi(\varphi)-$ $\Phi\left(\varphi^{\prime}\right)$ iterations. Each iteration applies up to two Stretch(.) operations, at $u$ or $v$ for some proper $\{u, v\}$ cut, and increases the number of vertices by at most 4 . By Lemma 2.15, the number of vertices increases by at most 4 times the decrease of the potential. Summation over all iterations of the while loop in Step (i) yields $N\left(\varphi_{1}\right) \leq N\left(\varphi_{0}\right)+4\left(\Phi\left(\varphi_{0}\right)-\Phi\left(\varphi_{1}\right)\right)$.

We can now focus on Steps (ii) (vii).

LEMma 2.16. Let $\varphi_{1}$ be an instance returned by Step (i). Then the while loop in Step (ii) terminates after at most $N_{\geq 3}(\varphi)$ iterations, and it returns an instance $\varphi_{2}$ such that $N\left(\varphi_{2}\right)<N\left(\varphi_{1}\right)+2 N_{\geq 3}\left(\varphi_{1}\right)$, $N_{\geq 3}\left(\varphi_{2}\right)=N_{\geq 3}\left(\varphi_{1}\right)$, and $\Phi\left(\varphi_{2}\right)=\Phi\left(\varphi_{1}\right)$.
Proof. Let $\varphi$ be an instance at the beginning of one iteration of the while loop in Step (ii) Since $\varphi$ is normal, every local graph $G_{\varphi}(\nu)$ is connected. Let $\{e, f\}$ be a proper 2-edge-cut in $G_{\varphi}(\nu)$, let $e=u_{1} v_{1}$ and $f=u_{2} v_{2}$ such both $v_{1}$ and $v_{2}$ are in a $\left\{u_{1}, u_{2}\right\}$ bridge $B$. Note that each component of $G_{\varphi}(\nu) \backslash\{e, f\}$ contains a vertex that has degree at least 3 in $G_{\varphi}(\nu)$, otherwise one of the components would be a path, and the 2-edge-cut would not be proper. In one iteration of Step (ii), an operation Enclose(.) replaces $G_{\varphi}(\nu)$ with two local graphs obtained by removing edges $e$ and $f$, and inserting two new paths $\left(u_{1}, w_{u}, u_{2}\right)$ and $\left(v_{1}, w_{v}, v_{2}\right)$, where $w_{u}$ and $w_{v}$ are new ordinary vertices of degree 2. In particular, $\Phi$ and the number of vertices of degree at least 3 do not change, and the total number of vertices in $\mathcal{G}$ increases by 2 .

It follows that $\Phi\left(\varphi_{2}\right)=\Phi\left(\varphi_{1}\right)$ and $N_{\geq 3}\left(\varphi_{2}\right)=$ $N_{\geq 3}\left(\varphi_{1}\right)$. Since each iteration in the while loop of Step (ii) increases the number of components of $\mathcal{G}$, but each new component contains at least one vertex of degree 3 or higher, the number of iterations is at most $N_{\geq 3}(\varphi)-1$. Summation over all iterations yields $N\left(\varphi_{2}\right) \leq N\left(\varphi_{1}\right)+2 N_{\geq 3}\left(\varphi_{1}\right)-2$.

Now we are ready to show that Subroutine 1 terminates.

Corollary 2.4. For an instance $\varphi_{0}$ of atomic embeddibility of size n, Subroutine 1 terminates.

Proof. By Corollary 2.3 the while loop in Step (i) terminates. By Lemma 2.16. Step (ii) terminates and eliminates all proper 2-edge-cuts containing an edge that is incident to a vertex of degree $\Delta=\Delta\left(\varphi_{0}\right)$. Finally, in the while loop of Step (iii), each iteration decreases the number of vertices of degree $\Delta$ in local graphs that are not p-stars. Therefore this while loop terminates, as well.

Note that Step (iii) increases the potential. We analyse the combined effect of Steps (iii) (vii), and show that they jointly decrease the potential, and we can charge the number of operations, as well as the number of new vertices to the decrease of the potential. The following observation will be helpful.

Lemma 2.17. Let $d$ and $d_{1}, \ldots, d_{k}$ be positive integers such that $d=\sum_{i=1}^{k} d_{i}, d \geq 4$, and $k \geq 2$. Then we have

$$
\begin{aligned}
(d-2)^{3} \geq & (2 d-5)+\sum_{i=1}^{k}\left(\max \left\{0, d_{i}-2\right\}\right)^{2}+ \\
& +\sum_{i=1}^{k}\left(\max \left\{0, d_{i}-2\right\}\right)^{3}
\end{aligned}
$$


with equality if and only if $k=d$, or $k=2$ and $\min \left\{d_{1}, d_{2}\right\}=1$.

Lemma 2.18. Consider Steps (iii) (vii) in an invocation of Subroutine 1 followed by Subroutine 2. We have $\Phi\left(\varphi_{2}\right)>\Phi\left(\varphi_{7}\right), N\left(\varphi_{7}\right) \leq N\left(\varphi_{2}\right)+O\left(\Phi\left(\varphi_{2}\right)-\Phi\left(\varphi_{7}\right)\right)$, and the number of operations performed in Steps (iii) (vii) is $O\left(\Phi\left(\varphi_{2}\right)-\Phi\left(\varphi_{7}\right)\right)$.

Proof. None of these steps increases the number of vertices of degree $\Delta$ or higher in local graphs. Ultimately all vertices of degree $\Delta$ outside of toroidal cycles are eliminated.

Overview. Each operation in Steps (iii) (vii) is associated to either a unique vertex of degree $\Delta$, or two virtual vertices of degree $\Delta$ that correspond to the same pipe. In Step (iii) and Steps (vi) (vii) this is vertex $v$; in Steps (iv) (v), these are virtual vertices $u$ and $v$ corresponding to the pipe $\mu \nu$. We consider each vertex $v$ of degree $\Delta$ in the instance $\varphi_{2}$, and analyse how the operations associated with $v$ change the potential and the total number of vertices over Steps (iii) (vii) Let $D(\Phi, v)$ and $D(N, v)$, resp., denote the changes in $\Phi($. and $N($.$) incurred by the operations associated with$ vertex $v$. We claim that for every vertex $v$ of degree $\Delta$ in $\mathcal{G}_{2}$, we have

$$
D(\Phi, v) \leq 0,
$$

with equality if and only if $v$ is a a local graph $G_{\varphi_{2}}(\nu)$ where $\nu$ is in a toroidal cycle; and

$$
D(N, v)+20 D(\Phi, v) \leq 0 .
$$

Note that 2.2 holds with a strict inequality for at least one vertex $v$. Indeed, we have $\Delta=\Delta\left(\varphi_{3}\right)=\Delta\left(\varphi_{2}\right)$, and so there is a vertex of degree $\Delta$ in some local graph of $\varphi_{2}$ outside of toroidal cycles. Summation over all vertices of degree $\Delta$ then yields

$$
\begin{aligned}
\Phi\left(\varphi_{7}\right) & =\Phi\left(\varphi_{2}\right)+\sum_{u \in V\left(\mathcal{G}_{2}\right): \operatorname{deg}(u)=\Delta} D(\Phi, u)<\Phi\left(\varphi_{2}\right), \\
N\left(\varphi_{7}\right) & =N\left(\varphi_{2}\right)+\sum_{u \in V\left(\mathcal{G}_{2}\right): \operatorname{deg}(u)=\Delta} D(N, u) \leq N\left(\varphi_{2}\right)+ \\
& +20\left(\Phi\left(\varphi_{2}\right)-\Phi\left(\varphi_{7}\right)\right) .
\end{aligned}
$$

Elimination of p-paths. Recall that each iteration of Step (iv.a) applies Contract $(\mu \nu)$ on a pipe $\mu \nu$ corresponding to virtual vertices $u$ in $G_{\varphi}(\mu)$ and $v$ in $G_{\varphi}(\nu)$. Without loss of generality, assume that $G_{\varphi}(\mu)$ is a p-path with poles $u$ and $w$. Operation Contract $(\mu \nu)$ eliminates $u$ and $v$, and creates a new local graph
$G_{\varphi}(\langle\mu \nu\rangle)$ where $G_{\varphi}^{-}(\langle\mu \nu\rangle)$ is isomorphic to $G_{\varphi}(\nu)$. For the analysis of $D(\Phi,$.$) and D(N,$.$) , we assume that this$ operation eliminates $u$ and $w$; and vertex $v$ of $G_{\varphi}(\nu)$ survives in $G_{\varphi}(\langle\mu \nu\rangle)$. Thus, the effect of Contract $(\mu \nu)$ is neutral for $v$, although $v$ may become an ordinary vertex if $w$ is ordinary before the operation.

Inequalities 2.2 -2.3 clearly hold for any vertex $v$ in toroidal cycles. For all other vertices of degree $\Delta$, we distinguish between three cases as follows.

Vertices of fixed rotation. Let $v$ be a vertex of fixed rotation with $\operatorname{deg}(v)=\Delta=\Delta\left(\varphi_{2}\right)$ in $\varphi_{2}$. If $v$ is an ordinary vertex, then Steps (i) (vi) do not change $v$, and in $\operatorname{Step~(vi)~a~} \operatorname{Stretch}(v,$.$) operation replaces v$ with two vertices $v_{1}$ and $v_{2}$, where $\operatorname{deg}\left(v_{1}\right)+\operatorname{deg}\left(v_{2}\right)=$ $\Delta+2$, and $\min \left\{\operatorname{deg}\left(v_{1}\right), \operatorname{deg}\left(v_{2}\right)\right\} \geq 3$. In this case, $D(\Phi, v)=\left(\operatorname{deg}\left(v_{1}\right)-3\right)+\left(\operatorname{deg}\left(v_{2}\right)-3\right)-(\Delta-3)=$ -1 , and $D(N, v)=1$. If $v$ is a virtual vertex, then $\operatorname{deg}(v)$ decreases in either Step (iv.b), (v.a) or (v.c), In Step (iv.b) or (v.a) one Stretch(.) operation has the same effect on the potential as for ordinary vertices, $D(\Phi, v)=-1$, but it creates two new vertices, and so $D(N, v)=2$. In Step (v.c) $\Delta-3$ successive Stretch(.) operations replace $v$ with $\Delta-2$ vertices of fixed orientation with degree 3. Thus, $D(\Phi, v)=$ $0-(\Delta-2)$, and $D(N, v) \leq \Delta-3$. In all cases, 2.2 2.3 follow.

Poles of p-paths. Let $v$ be a pole of a p-path $G_{\varphi_{2}}(\nu)$, with $\operatorname{deg}(u)=\Delta=\Delta\left(\varphi_{2}\right)$. Denote the other pole of the p-path by $u$, where obviously $\operatorname{deg}(u)=$ $\operatorname{deg}(v)=\Delta$. If both $u$ and $v$ are ordinary, then Steps (i) (v) do not change $G_{\varphi_{2}}(\nu)$. The Detach(.) operation in Step (vii) replaces $v$ with $\Delta$ new vertices of degree 1. Thus, $D(\Phi, v)=-(\Delta-2)^{2}$ and $D(N, v)=$ $\Delta-1$, thus (2.2)-2.3) follow.

Assume that $u$ or $v$ is a virtual vertex. Then a Contract(.) operation in Step (iv.a) eliminates both $u$ and $v$. We have $D(\Phi, u)=-(\Delta-2)^{2}$ and $D(N, u)=$ -1 .

Step (iv.b) applies Stretch(.) to a pair of virtual vertices $u_{1}$ and $u_{2}$ of $G_{\varphi}(\mu)$. Thus, we have $D\left(\Phi, u_{1}\right)=$ $D\left(\Phi, u_{2}\right)<0$ and $D\left(N, u_{1}\right)=D\left(N, u_{2}\right)=1$.

Proper cut vertices. Let $v$ be a proper cut vertex in $\varphi_{2}$ with $\operatorname{deg}(v)=\Delta=\Delta\left(\varphi_{2}\right)$ in some local graph $G_{\varphi_{2}}(\nu)$. Assume $v$ has $k \geq 2$ bridges $B_{1}, \ldots, B_{k}$, and $\operatorname{deg}_{B_{i}}(v)=d_{i}$ for all $i \in[k]$. Step (iii) successively encloses the $k$ bridges. Note that $v$ remains a proper cut vertex of degree $\Delta$. Step (iii) creates new virtual vertices $v_{1}, \ldots, v_{k}$ in the $\mathrm{p}$-star centered at $v$, where $\operatorname{deg}(v)=\sum_{i=1}^{k} \operatorname{deg}\left(v_{i}\right)$. Every new virtual vertex $v_{i}$, $i \in[k]$, is part of a proper 2-cut $\left\{v, v_{i}\right\}$.

Moreover, every virtual vertex $v_{i}, i \in[k]$, corresponds to another virtual vertex $v_{i}^{\prime}$ in the local graph of an atom created by enclosing $B_{i}$; this local graph 
is isomorphic to $B_{i}$, where $v_{i}^{\prime}$ plays the role of $v$. In particular $v_{i}^{\prime}$ cannot be a cut vertex, but it may be contained in a proper 2-cut. At the end of Step (iii), we obtain a $\Delta$-nice instance $\varphi_{3}$ in which none of the local graphs containing a virtual vertex $v_{i}^{\prime}, i \in[k]$, is a p-path or a p-star contain any vertex of degree $\Delta$. Therefore Steps (iv) (vii) do not change the degree of $v_{i}^{\prime}$, and $v_{i}^{\prime}$ cannot become a cut vertex for any $i \in[k]$.

Next we consider the possible changes to the pstar centered at $v$ in Steps (iv) (vii) Step (iv.a) may turn $v$ into an ordinary vertex as noted above (but it changes neither $D(\Phi, v)$ nor $D(N, v))$. Since $v$ is a proper cut vertex of degree $\Delta$, the next step that can possibly modify the p-star is Step (v.b) (v.c), or (vii) In Step (v.b), a Contract(.) operation eliminates vertex $v$, and any vertex $v_{i}, i \in[k]$ may become a cut vertex. In Step (v.c), successive Stretch(.) operations replace $v$ with $\Delta-2$ vertices of degree 3 . Since each of these vertices could be a proper cut vertex, they contribute $(\Delta-2)(3-2)^{3}=\Delta-2$ to the potential. Finally, if $v$ is an ordinary vertex, then $\operatorname{Detach}(v)$ in Step (vii) replaces $v$ with $\Delta$ vertices of degree 1 , which do not contribute to the potential.

At the beginning of Step (iii), vertex $v$ contributes $(\Delta-2)^{3}$ to $\Phi\left(\varphi_{2}\right)$. At the end of Subroutine 2 , the contribution of $v$, together with the virtual vertices $v_{i}$ and $v_{i}^{\prime}$, over all $i \in[k]$, is at most

$$
(\Delta-2)+\sum_{i=1}^{k}\left(\max \left\{0, d_{i}\right\}\right)^{3}+\sum_{i=1}^{k}\left(\max \left\{0, d_{i}\right\}\right)^{2} .
$$

By Lemma 2.17, $D(\Phi, v) \leq-(\Delta-3) \leq-\Delta / 4$.

Let us estimate the number of new vertices created in these steps. In Step (iii), the Enclose(.) operations create a pair of virtual vertices for each bridge of $v$ (i.e., $2 k$ vertices), and up to $k$ ordinary subdivision vertices. In Step (v.c) Stretch(.) operations create $\Delta-2$ new vertices; and in Step (vii) the $\operatorname{Detach}(v)$ operation increases the number of vertices by $\Delta-1$. Therefore, $D(N, v) \leq 3 k+2 \Delta-3 \leq 5 \Delta-3$. Since $D(\Phi, v) \leq-\Delta / 4$, inequalities $(2.2)$ and $(2.3)$ follow.

Lemma 2.19. For an instance $\varphi_{0}$ of atomic embeddability of size $n$, Algorithm terminates, it performs $O\left(N_{>3}\left(\varphi_{0}\right)+\Phi\left(\varphi_{0}\right)\right)$ operations, and runs in $O\left(n^{8}\right)$ time ${ }^{3}$

Proof. Consider one iteration of the while loop of Step (I), which calls Subroutines 1 and 2. By Corollary 2.3. Step (i) terminates, performs $O\left(\Phi\left(\varphi_{0}\right)-\Phi\left(\varphi_{1}\right)\right)$

\footnotetext{
${ }^{3}$ Optimizing the running time analysis further, which we believe is possible, is beyond the scope of this work.
}

operations, and returns and instance $\varphi_{1}$ with and $N\left(\varphi_{1}\right) \leq N\left(\varphi_{0}\right)+12\left(\Phi\left(\varphi_{0}\right)-\Phi\left(\varphi_{1}\right)\right)$. By Lemma 2.16 . Step (ii) terminates, performs $O\left(N_{\geq 3}\left(\varphi_{0}\right)\right)$ operations, and returns an instance $\varphi_{2}$ with $N_{\geq 3}\left(\varphi_{2}\right)=N_{\geq 3}\left(\varphi_{1}\right)$ and $\Phi\left(\varphi_{2}\right)=\Phi\left(\varphi_{1}\right)$. Similarly, by Lemma 2.18 . the sequence of Steps (iii) (vii) terminates, performs $O\left(\Phi\left(\varphi_{2}\right)-\Phi\left(\varphi_{7}\right)\right)$ operations, and returns an instance $\varphi_{7}$ with $N\left(\varphi_{7}\right) \leq N\left(\varphi_{2}\right)+O\left(\Phi\left(\varphi_{2}\right)-\Phi\left(\varphi_{7}\right)\right)$.

Using the definition of the potential, we can bound its initial value by

$$
\begin{aligned}
\Phi\left(\varphi_{0}\right) & =\sum_{v \in V\left(\mathcal{G}_{0}\right)}(\max \{0, \operatorname{deg}(v)-\xi(v)\})^{\sigma(v)} \leq \\
& \leq n \cdot\left(\Delta\left(\varphi_{0}\right)-2\right)^{3} \leq O\left(n^{4}\right) .
\end{aligned}
$$

The while loop in Step (I) of the Algorithm terminates after $\Delta\left(\varphi_{0}\right)-3 \leq n$ iterations, since each iteration decreases $\Delta($.$) by Lemmas 2.11$ and 2.13 In each iteration, the potential $\Phi($.$) decreases, and N($.$) increases by$ at most constant times the decrease of the potential by Lemmas 2.15 2.16 and 2.18. In particular, for every instance $\varphi^{*}$ in intermediate phases of Step (I) both $N\left(\varphi^{*}\right)$ and $N_{\geq 3}\left(\varphi^{*}\right)$ are bounded by $O\left(n+\Phi\left(\varphi_{0}\right)\right) \leq O\left(n^{4}\right)$.

Each operation in Steps (i) (vii) can be implemented in $O\left(N\left(\varphi^{*}\right)\right)$ time, where $\varphi^{*}$ is the instance for which the operation is applied (this allows for planarity testing, and recomputing block trees and SPQR-trees after each operation). As noted above, we have $N\left(\varphi^{*}\right) \leq$ $O\left(n+\Phi\left(\varphi_{0}\right)\right) \leq O\left(n^{4}\right)$. The overall running time of all invocations of Step (i) (vii) is $O\left(n^{4}\left(n+\Phi\left(\varphi_{0}\right)\right)\right) \leq$ $O\left(n^{8}\right)$.

By Lemmas 2.9 and 2.10. Steps (II) (III) of the Algorithm run in $O\left(N\left(\varphi_{7}\right)\right) \leq O\left(n+\Phi\left(\varphi_{0}\right)\right) \leq O\left(n^{4}\right)$ time.

THEOREM 2.1. There is an algorithm that determines whether a simplicial map $\varphi: G \rightarrow H$ is atomic embeddable in time polynomial in the number of edges and vertices in $G$ and $H$.

Proof. Let $\varphi$ be an instance $\varphi$ of atomic embeddability of size $n$, where $n$ is the number of edges and vertices in $G$ and $H$. The Preprocessing algorithm runs in $O(n)$ time and returns an equivalent normal instance $\varphi_{0}$ of size $O(n)$ by Lemma 2.3. The main Algorithm for $\varphi_{0}$ terminates in $O\left(n^{8}\right)$ time by Lemma 2.19, and determines whether $\varphi_{0}$ is atomic embeddable by Lemma 2.14 . Since $\varphi_{0}$ and $\varphi$ are equivalent, this also determines whether $\varphi$ is atomic embeddable.

\section{Beyond Atomic Embeddings}

Since atomic embeddability is tractable, it makes sense to consider its generalizations in which every atom can have genus higher than 0 . 


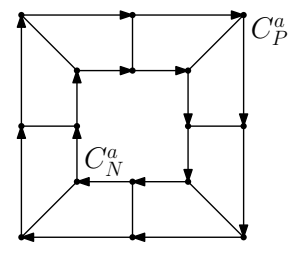

Figure 13: The variable gadget $G_{0}^{a}=C^{a} \square P_{1}$ for the NP-hardness reduction of Not-All-Equal 3SAT to compatible toroidal embeddability.

We consider the GENERALIZED ATOMIC EMBEDDING problem for a simplical map $\varphi: G \rightarrow H$, where $G$ and $H$ are multigraphs without loops. The only difference from ATOMIC EMBEDDIBILITY is that we define the surface $\mathcal{H}$ as follows: For each atom $\nu \in V(H)$, we construct $\mathcal{S}(\nu)$ from an oriented surface of genus $g(\nu)$ without boundary (rather than a 2-sphere), and remove $\operatorname{deg}(\nu)$ holes. Hence, an instance for generalized atomic embeddability is a pair $(\varphi, g)$, where $\varphi$ is a simplicial map $\varphi: G \rightarrow H$ and $g: V(H) \rightarrow \mathbb{N}_{0}$.

Problem 4. (Generalized atomic embeddability) Given a simplicial map $\varphi: G \rightarrow H$, where $G$ and $H$ are multigraphs without loops, and a function $g: V(H) \rightarrow \mathbb{N}_{0}$, decide whether a generalized atomic embedding of $G$ with respect to $\varphi$ exists.

In this section, we show that GENERALIZED ATOMIC EMBEDDABILITY is NP-hard, and therefore also NPcomplete, even when $g(\nu) \leq 1$ for all atoms $\nu \in V(H)$, and the number of vertices in $\varphi^{-1}[\nu]$ is at most 7 for each atom $\nu$ with $g(\nu)=1$.

The NP-hardness proof is based the embeddings of $K_{3,4}$ on a torus. For an embedding of $K_{3,4}$ on a torus, we say that two vertices $u$ and $v$ of the same vertex class (i.e., with the same degree) have the same rotation if the rotation of $u$ is $\left(u v_{1}, \ldots, u v_{k}\right)$ and the rotation at $v$ is $\left(v v_{1}, \ldots, v v_{k}\right)$, where $\left\{v_{1}, \ldots, v_{k}\right\}$ is a vertex class of $K_{3,4}$. The following lemma extends a lemma from a paper of Kynčl and the first author [24, Theorem 7(b)].

LEMMA 3.1. In every embedding of $K_{3,4}$ on the torus the four cubic vertices do not all have the same rotation; subject to the previous claim, any rotations for the four cubic vertices can be realized by a toroidal embedding.

Theorem 3.1. Generalized Atomic Embeddability is NP-hard.

Proof. We reduce Generalized Atomic EmbedDABILITY from Not-ALL-EQUAL 3SAT, which is known to be NP-complete. An instance of NoT-ALLEQUAL 3 SAT is given by a pair $(\mathcal{A}, \mathcal{C})$, where $\mathcal{A}$ is a finite set of boolean variables and $\mathcal{C}$ is a finite set of clauses, each of which is a conjunction of three literals. Each literal is either a variable $a \in \mathcal{A}$ or the negation of $a$, denoted by $\neg a$. An instance $(\mathcal{A}, \mathcal{C})$ is positive if there exists an assignment $\tau: \mathcal{A} \rightarrow\{$ true, false $\}$ such that at least 1 and at most 2 literals are true in every clause.

Given an instance $(\mathcal{A}, \mathcal{C})$ of Not-AlL-EQUAL 3SAT, we construct an instance $(\varphi, g)$ for GEnERALIZED ATOMIC EMBEDDABILITY, and show that it is positive if and only if $(\mathcal{A}, \mathcal{C})$ is positive. Let $\mathcal{C}=$ $\left\{C_{1}, \ldots, C_{n}\right\}$.

The construct an instance $(\varphi, g)$, where $\varphi: G \rightarrow H$, $g: V(H) \rightarrow \mathbb{N}_{0}$. Let the multigraph $H$ be a p-star with a center $\nu_{0}$, and $n$ additional atoms $\nu_{1}, \ldots, \nu_{n}$, such that there are 6 pipes between $\nu_{0}$ and each $\nu_{i}$, for $i=1, \ldots, n$. In particular, all pipes are incident to the center $\nu_{0}$.

We put $g\left(\nu_{0}\right)=0$ and $g\left(\nu_{1}\right)=\ldots=g\left(\nu_{n}\right)=$ 1. We describe $\varphi$ via a construction of local graphs $G_{0}=G_{\varphi}\left(\nu_{0}\right), G_{1}=G_{\varphi}\left(\nu_{1}\right), \ldots, G_{n}=G_{\varphi}\left(\nu_{n}\right)$. For ease of presentation, we describe the local graphs as semi-directed graphs (in which some edges are directed and others are undirected). However, in the eventual instance $(\varphi, g)$, all local graphs are undirected (by replacing every directed edge with an undirected edge.)

The local graphs $G_{1}, \ldots, G_{n}$ are each isomorphic to $K_{3,4}$. Let $G_{0}$ be a disjoint union of the semi-directed graphs $G_{0}^{a}=C^{a} \square P_{1}$, for all $a \in \mathcal{A}$, where $P_{1}$ is a path of length 1 and $C^{a}$ is a directed cycle whose length is equal to four times the number of occurrences of $a$ in the clauses in $\mathcal{C}$. Let $C_{P}^{a}$ and $C_{N}^{a}$ denote the two vertex disjoint directed induced cycles in $G_{0}^{a}$ of length equal to the length of $C^{a}$, whose orientation is inherited from $C^{a}$; see Fig. 13 for an illustration.

Next, we define the pipes in $E(H)$ by designating the pairs of corresponding virtual vertices in the local graphs; furthermore, for if a pipe $\rho \in E(H)$ corresponds to virtual vertices $u$ and $v$ in two local graphs, we also specify a bijection between the set of edges incident to $u$ and the set of edges incident to $v$. All vertices in local graphs that are not designated to be virtual will be ordinary. This uniquely determines the instance $(\varphi, g)$.

For each clause $C_{i} \in \mathcal{C}, i \in[n]$, we define three pipes in $E(H)$. Assume that $C_{i}=\left(\ell_{1} \vee \ell_{2} \vee \ell_{3}\right)$. Recall that $G_{i}$ is isomorphic to $K_{3,4}$. Label the three vertices of degree 4 in $G_{i}$ by $-1,0$, and 1 resp.; two arbitrary cubic vertices by the literal $\ell_{1}$; and the remaining two cubic vertices by the literals $\ell_{2}$ and $\ell_{3}$, respectively. Let every cubic vertex $v \in V\left(G_{i}\right)$ be virtual, and let each vertex with label $\ell \in\left\{\ell_{1}, \ell_{2}, \ell_{3}\right\}$ correspond a vertex $u$ in $C_{P}^{a}$ if $\ell=a$ and a vertex $u$ in $C_{N}^{a}$ if $\ell=\neg a$. We construct the bijection between the edges incident to $v$ 
and the edges incident to $u$ as follows: Let the edge between $v$ and the vertex of $G_{i}$ with label $-1,0$, and 1 , resp., correspond to the incoming, undirected, and outgoing edge incident to $v^{\prime}$ in $G_{0}$. This completes the definition of the instance $(\varphi, g)$

It remains to prove that $(\varphi, g)$ is a positive instance if and only if $(\mathcal{A}, \mathcal{C})$ is a positive instance. We give the proof only for the forward direction. Assume that $(\varphi, g)$ is a positive instance of Generalized ATOMIC EMBEDDIBILITY. Let $\mathcal{E}: G \rightarrow \mathcal{H}$ be a generalized atomic embedding of $G$ with respect to $(\varphi, g)$. Since $g\left(\nu_{0}\right)=0$, the restriction of $\mathcal{E}$ on $\mathcal{S}\left(\nu_{0}\right)$ yields an embedding of $G_{0}$ in the plane; and an embedding of $G_{i}$ in the torus for all $i \in[n]$. We construct a satisfying assignment $\tau: \mathcal{A} \rightarrow\{$ true, false $\}$ based on the embedding of $G_{0}$ as follows. We put $\tau(a)=$ true if the incoming, undirected, and outgoing edges incident to a vertex $v$ in $C_{P}^{a}$ appear in this counterclockwise order in the rotation of $v$ in the embedding of $G_{0}$; and we put $\tau(a)=$ false otherwise. Note that the truth value of $a$ is independent of the choice of $v$. Also note that a literal $\ell$ of the clause $C_{i}, i \in[n]$, is satisfied if and only if the edges between the vertex $v$ labeled by $\ell$ and the vertices labeled by $-1,0$ and 1 in $G_{i}$ appear in this clockwise order in the rotation at $v$ in the embedding of $G_{i}$. Note that Observation 1 holds also for generalized atomic embeddability. By Lemma 3.1 and Observation 1, every clause $C_{i}$ must be satisfied by at least 1 and at most 2 literals, and hence, $(\mathcal{A}, \mathcal{C})$ is positive.

\section{C-planarity and Connected SEFE-2}

The aim of this section is to given a polynomial-time reduction of C-PLANARITY, Problem 1, as well as a special case of a related problem of SEFE-2 (defined below) to thickenability, Problem 2

An instance of the c-planarity problem is given by the triple $(G, \mathcal{C}, \mu)$, where $G, \mathcal{C}$, and $\mu$ are as in the input to the problem. The instance is positive if its output answer is True. The embedding $\psi$ of $G$ in $\mathbb{R}^{2}$ (or on a 2-sphere) such that $\psi(v) \in \mu(v)$ and $|\psi(e) \cap C| \leq 1$, for every $C \in \mathcal{C}$, is c-planar. Cortese and Partignani [19. Theorems 1 and 2] showed that in order to solve the c-planarity problem it is enough to concentrate on the special of c-planarity of so-called INDEPENDENT FLAT CLUSTERED PLANARITY in which curves in $\mathcal{C}$ bound pairwise disjoint disks, $\mu^{-1}[R]$ induces an independent set for every $R \in \mathcal{R}$, and $\mu^{-1}[R]$ is empty for the unbounded region $R$. The following theorem allows us to consider only instances of independent flat clustered planarity in the reduction.

TheOrem 4.1. ([19]) C-Planarity is polynomialtime equivalent to INDEPENDENT FLAT CLUSTERED
PLANARITY.

Given an instance $(G, \mathcal{C}, \mu)$ of InDEPENDENT FLAT CLUSTERED PLANARITY, for convenience, we subdivide every edge of $G$ by a single vertex which is mapped by $\mu$ to the unbounded region. Clearly, this modification does not change whether the instance is positive or negative. By a slight abuse of notation, we denote the resulting subdivided instance by $(G, \mathcal{C}, \mu)$. We say that $(G, \mathcal{C}, \mu)$ is subdivided independent flat.

Also we label the bounded regions of $\mathbb{R}^{2} \backslash \bigcup_{C \in \mathcal{C}} C$ by their bounding curves. Note that every edge of $G$ has exactly one of its vertices mapped by $\mu$ to the unbounded region.

Thickenability. In the following, we express THICKENABILITY as a combinatorial problem, to which we actually reduce C-PLANARITY.

Let $P=(H, F)$ be a 2-polyhera. Let $F=$ $\left\{f_{1}, \ldots, f_{|F|}\right\}$. A 2-polyhedron $P$ embeds in an orientable 3 -manifold $M$ (such as $\mathbb{R}^{3}$ ) if the following holds. We can embed $H$ in $M$ so that we can represent facets $f_{1}, \ldots, f_{|T|}$ by pairwise interior disjoint topological discs $D_{1}, \ldots, D_{|T|}$, resp., in $M$ such that for every $i \in[|F|]$ the boundary of $D_{i}$, denoted by $\partial D_{i}$, consists of the embedded cycle $f_{i}$. The representation of $P$ in $M$ given by the discs $D_{1}, \ldots, D_{|F|}$ is an embedding of $P$. The restriction of the embedding of $P$ to the boundaries of these discs gives the embedding of $H$.

For $v \in V(H)$, the link of $v$ in $P$ is a multigraph $L_{P}(v)=(E(v), F(v))$, where $E(v)$ is the multiset of edges in $H$ incident to $v$, and $F(v)$ is in a bijection with the set of facets in $P$ that are incident to $v$ and we give it next. A pair $e \in E(v)$ and $g \in E(v)$ is joined by as many edges in $F(v)$ as there are facets in $F$ containing $e$ and $g$.

If $P=(H, F)$ is thickenable then the intersection of its embedding in a manifold with a sufficiently small 2-sphere centered at (the embedding of) a vertex $v \in V(H)$ is a spherical embedding of the link $L_{P}(v)$. Indeed, the 2-sphere intersects edges of $H$ incident to $v$ in points and facets in $F$ incident to $v$ in curves between these points.

Given $P=(H, F)$, the family $\left\{\mathcal{E}_{v} \mid v \in V(H)\right\}$, where $\mathcal{E}_{v}$ is a planar (spherical) embedding of $L_{P}(v)$, is compatible, if for every $e \in E(H)$ joining vertices $u$ and $v$ it holds that the rotation at $e$ in $\mathcal{E}_{u}$ is opposite to the rotation at $e$ in $\mathcal{E}_{v}$. The observation from the previous paragraph proves the "only if" part of the theorem of Neuwirth 37 .

THEOREM 4.2. (37]) The 2-dimensional polyhedron $P=(H, F)$ is thickenable if and only if there exists a family of compatible embeddings of the vertex links of $H$. 
We show that testing the condition of Theorem 4.2 References

generalizes C-PLANARITY and CONNECTED SEFE- 2 .

Reduction. In what follows we define a 2dimensional polyhedron that is thickenable if and only if $(G, \mathcal{C}, \mu)$ is positive. Let $P=P(G, \mathcal{C}, \mu)=(H, T)$ denote the 2-dimensional polyhedron, where $H$ and $T$ are defined as follows. (The letter $T$ indicates that all the facets are triangles.) The vertex set $V(H)$ of $H$ is $\mathcal{C} \cup\{R\} \cup\{A\}$, where $R$ is the unbounded region of $\mathbb{R}^{2} \backslash \bigcup_{C \in \mathcal{C}} C$. The multiset $E(H)$ of edges in $H$ is in a bijection with $V(G) \cup \mathcal{C}$, and we give it next. The vertex $R$ is joined with every $C \in \mathcal{C}$ by a single edge, and for every $v \in V(G)$, we add an edge $e(v)$ joining $\mu(v)$ and A. Finally, $T=\{\{\mu(u) \mu(v), e(u), e(v)\} \mid u v \in E(G)\}$, and hence, the set of triangular facets $T$ is in a bijection with $E(G)$. The definition of $T$ is consistent with the definition of $H$, since every edge of $G$ has exactly one of its vertices mapped by $\mu$ to $R$.

It remains to prove that the polyhedron $P$ has the desired property.

Lemma 4.1. The 2-dimensional polyhedron $P=$ $P(G, \mathcal{C}, \mu)$ is thickenable if and only if $(G, \mathcal{C}, \mu)$ is a positive instance of C-PLANARITY.

An immediate consequence of Lemma 4.1 is the main result of this section.

Theorem 4.3. C-Planarity reduces to thickenABILITY in polynomial time.

Simultaneous embedding with fixed edges (SEFE-2). In the following we discuss an implication of Theorem 4.3 to the problem of simultaneous embeddability of two graphs SEFE-2, which is formally described as follows.

Problem 5. Sefe-2. Given two (planar) graphs, $G_{1}=$ $\left(V, E_{1}\right)$ and $G_{2}=\left(V, E_{2}\right)$, decide whether there exists a planar embedding $\mathcal{E}$ of $G=G_{1} \cup G_{2}$ such that both $\mathcal{E}\left[G_{1}\right]$ and $\mathcal{E}\left[G_{2}\right]$ are embeddings.

The CONNECTED SEFE-2 is a special case of SEFE-2 in which $G_{1} \cap G_{2}$ is connected. Angelini and Da Lozzo 4 showed that CONNECTED SEFE-2 is polynomial-time equivalent to C-PLANARITY. Together with Theorem 4.3 , this immediately implies the following.

Corollary 4.1. Connected Sefe-2 reduces in polynomial time to THICKENABILITY.

\section{Acknowledgment}

The first author would like to thank Arnaud de Mesmay for pointing him to Johannes Carmesin's work. Thanks are extended to Johannes Carmesin for kindly discussing this work with him.

[1] Hugo A. Akitaya, Greg Aloupis, Jeff Erickson, and Csaba D. Tóth. Recognizing weakly simple polygons. Discrete \&6 Computational Geometry, 58(4):785-821, 2017. doi:10.1007/s00454-017-9918-3.

[2] Hugo A. Akitaya, Radoslav Fulek, and Csaba D. Tóth. Recognizing weak embeddings of graphs. ACM Trans. Algorithms, 15(4):50:1-50:27, 2019. doi:10. 1145/3344549.

[3] Patrizio Angelini, Giuseppe Di Battista, Fabrizio Frati, Maurizio Patrignani, and Ignaz Rutter. Testing the simultaneous embeddability of two graphs whose intersection is a biconnected or a connected graph. $J$. Discrete Algorithms, 14:150-172, 2012. doi:doi.org/ 10.1016/j.jda.2011.12.015

[4] Patrizio Angelini and Giordano Da Lozzo. SEFE = C-Planarity? Comput. J., 59(12):1831-1838, 2016. doi:10.1093/comjnl/bxw035.

[5] Patrizio Angelini and Giordano Da Lozzo. Clustered planarity with pipes. Algorithmica, 81(6):2484-2526, 2019. doi:10.1007/s00453-018-00541-w

[6] S. B. Belyi. Self-nonintersecting and non intersecting chains. Mathematical notes of the Academy of Sciences of the USSR, 34(4):802-804, 1983. Translated from Matematicheskie Zametki, 34(4):625-628, 1983. doi: 10.1007/BF01157400.

[7] Thomas Bläsius, Annette Karrer, and Ignaz Rutter. Simultaneous embedding: Edge orderings, relative positions, cutvertices. Algorithmica, 80(4):1214-1277, 2018. doi:10.1007/s00453-017-0301-9.

[8] Thomas Bläsius, Stephen G. Kobourov, and Ignaz Rutter. Simultaneous embedding of planar graphs. In Handbook of Graph Drawing and Visualization. Chapman and Hall/CRC, 2013.

[9] Thomas Bläsius and Ignaz Rutter. Simultaneous PQordering with applications to constrained embedding problems. ACM Trans. Algorithms, 12(2):article 16, 2016. doi:10.1145/2738054

[10] Peter Brass, Eowyn Cenek, Cristian A. Duncan, Alon Efrat, Cesim Erten, Dan P. Ismailescu, Stephen G. Kobourov, Anna Lubiw, and Joseph S.B. Mitchell. On simultaneous planar graph embeddings. Computational Geometry, 36(2):117-130, 2007. doi:doi.org/ 10.1016/j.comgeo.2006.05.006

[11] Benjamin A. Burton, Arnaud de Mesmay, and Uli Wagner. Embeddability of 2-complexes. In Benjamin Burton, Herbert Edelsbrunner, Jeff Erickson, and Stephan Tillmann, editors, Computational Geometric and Algebraic Topology, Oberwolfach Reports, chapter 45, pages 2666-2668. 2015. doi:10.14760/OWR-2015-45.

[12] Benjamin A Burton, Arnaud de Mesmay, and Uli Wagner. Finding non-orientable surfaces in 3-manifolds. Discrete \& Computational Geometry, 58(4):871-888, 2017. doi:10.1007/s00454-017-9900-0.

[13] Johannes Carmesin. Embedding simply connected 2complexes in 3-space - II. rotation systems. Preprint, arXiv:1709.04643, 2017. 
[14] Johannes Carmesin. Embedding simply connected 2complexes in 3-space - V. A refined Kuratowski-type characterisation. Preprint, arXiv:1709.04659, 2017.

[15] Hsien-Chih Chang, Jeff Erickson, and Chao Xu. Detecting weakly simple polygons. In Proc. 26th $A C M$ SIAM Symposium on Discrete Algorithms (SODA), pages 1655-1670. SIAM, 2015. doi:10.1137/1. 9781611973730.110

[16] Pier Francesco Cortese and Giuseppe Di Battista. Clustered planarity (invited lecture). In Proc. 21st Symposium on Computational Geometry (SoCG), pages 30-32. ACM Press, 2005. doi:10.1145/ 1064092.1064093

[17] Pier Francesco Cortese, Giuseppe Di Battista, Fabrizio Frati, Maurizio Patrignani, and Maurizio Pizzonia. Cplanarity of c-connected clustered graphs. J. Graph Algorithms Appl., 12(2):225-262, 2008. doi:10.7155/ jgaa.00165.

[18] Pier Francesco Cortese, Giuseppe Di Battista, Maurizio Patrignani, and Maurizio Pizzonia. On embedding a cycle in a plane graph. Discrete Mathematics, 309(7):1856-1869, 2009.

[19] Pier Francesco Cortese and Maurizio Patrignani. Clustered planarity $=$ flat clustered planarity. In Proc. 26th Symposium on Graph Drawing and Network Visualization (GD), pages 23-38, 2018. doi:10.1007/ 978-3-030-04414-5_2

[20] Arnaud de Mesmay, Yo'av Rieck, Eric Sedgwick, and Martin Tancer. Embeddability in $\mathbb{R}^{3}$ is NP-hard. In Proc. 29th ACM-SIAM Symposium on Discrete Algorithms (SODA), pages 1316-1329, 2018. doi: 10.1137/1.9781611975031.86

[21] Giuseppe Di Battista and Roberto Tamassia. Incremental planarity testing. In 30th IEEE Symposium on Foundations of Computer Science (FOCS), pages 436441, 1989. doi:10.1109/SFCS.1989.63515

[22] Qing-Wen Feng, Robert F. Cohen, and Peter Eades. How to draw a planar clustered graph. In Proc. 1st Conference on Computing and Combinatorics (COCOON), volume 959 of $L N C S$, pages 21-30. Springer, Berlin, 1995. doi:10.1007/BFb0030816

[23] Qing-Wen Feng, Robert F. Cohen, and Peter Eades. Planarity for clustered graphs. In Proc. 3rd European Symposium on Algorithms (ESA), volume 979 of $L N C S$, pages 213-226. Springer, 1995. doi:10.1007/ 3-540-60313-1_145.

[24] Radoslav Fulek and Jan Kynčl. Counterexample to an extension of the Hanani-Tutte theorem on the surface of genus 4. Preprint, arXiv:1709.00508, 2017.

[25] Radoslav Fulek and Jan Kynčl. Hanani-Tutte for approximating maps of graphs. Preprint, arXiv:1705.05243, 2017.

[26] Radoslav Fulek and Jan Kynčl. Hanani-Tutte for approximating maps of graphs. In Proc. 34th Symposium on Computational Geometry (SoCG), volume 99 of LIPIcs, pages 39:1-39:15. Schloss Dagstuhl, 2018. doi:10.4230/LIPICs.SoCG.2018.39

[27] Radoslav Fulek, Jan Kynčl, Igor Malinović, and
Dömötör Pálvölgyi. Clustered planarity testing revisited. Electron. J. Combin., 22(4):article P4.24, 2015. URL: https://www.combinatorics.org/ojs/ index.php/eljc/article/view/v22i4p24

[28] Carsten Gutwenger, Michael Jünger, Sebastian Leipert, Petra Mutzel, Merijam Percan, and René Weiskircher. Advances in c-planarity testing of clustered graphs. In Proc. 10th Symposium on Graph Drawing, volume 2528 of LNCS, pages 220-236. Springer, 2002. doi:10.1007/3-540-36151-0_21

[29] Bernhard Haeupler, Krishnam Raju Jampani, and Anna Lubiw. Testing simultaneous planarity when the common graph is 2-connected. J. Graph Algorithms Appl., 17(3):147-171, 2013. doi:10.7155/jgaa.00289

[30] Allen Hatcher. Algebraic topology. Cambridge University Press, 2005.

[31] John E. Hopcroft and Robert E. Tarjan. Efficient planarity testing. J. ACM, 21(4):549-568, 1974. doi: $10.1145 / 321850.321852$.

[32] Thomas Lengauer. Hierarchical planarity testing algorithms. J. ACM, 36(3):474-509, 1989. doi:10.1145/ 65950.65952

[33] Saunders Mac Lane. A structural characterization of planar combinatorial graphs. Duke Math. J., 3(3):460472, 1937. doi:10.1215/S0012-7094-37-00336-3

[34] Jiří Matoušek, Eric Sedgwick, Martin Tancer, and Uli Wagner. Embeddability in the 3-sphere is decidable. J. ACM, 65(1):5:1-5:49, 2018. doi:10.1145/3078632

[35] Arnaud De Mesmay, Vojtěch Kaluža, and Martin Tancer, 2019. Personal communication.

[36] Piotr Minc. On simplicial maps and chainable continua. Topology and its Applications, 57(1):1-21, 1994. doi:10.1016/0166-8641(94)90028-0

[37] Lee Neuwirth. An algorithm for the construction of 3manifolds from 2-complexes. Mathematical Proceedings of the Cambridge Philosophical Society, 64(3), 1968. doi:10.1017/S0305004100043279.

[38] Dušan Repovš and Arkadij B. Skopenkov. A deleted product criterion for approximability of maps by embeddings. Topology Appl., 87(1):1-19, 1998. doi: 10.1016/S0166-8641(97)00121-1

[39] Marcus Schaefer. Toward a theory of planarity: Hanani-Tutte and planarity variants. J. Graph Algorithms Appl., 17(4):367-440, 2013. doi:10.7155/ jgaa.00298

[40] Karol Sieklucki. Realization of mappings. Fundamenta Mathematicae, 65(3):325-343, 1969. doi:10.4064/ fm-65-3-325-343.

[41] Arkadiy B. Skopenkov. A generalization of Neuwirth's theorem on thickening 2-dimensional polyhedra. Mathematical Notes, 58(5):1244-1247, 1995. doi:10.1007/ BF02305012

[42] Mikhail Skopenkov. On approximability by embeddings of cycles in the plane. Topology Appl., 134(1):122, 2003. doi:10.1016/S0166-8641(03)00069-5 